\title{
CONCEPTUAL AERODYNAMIC DESIGN OF A TAIL-CONE THRUSTER SYSTEM UNDER AXI-SYMMETRIC INLET DISTORTION
}

\author{
B.J. Lee \\ Vantage Partners, LLC \\ Turbomachinery and Turboelectric Systems Branch \\ NASA Glenn Research Center \\ Cleveland, OH, USA
}

\author{
May-Fun Liou \\ Inlet and Nozzle Branch \\ NASA Glenn Research Center \\ Cleveland, $\mathrm{OH}, \mathrm{USA}$
}

\author{
Meng-Sing Liou* \\ Propulsion Division \\ NASA Glenn Research Center \\ Cleveland, OH, USA
}

\begin{abstract}
This paper presents a conceptual design of a tail-cone thruster system which is operating under an axisymmetric inlet distortion. An effort to realize the targeted fuel burn saving that was proposed in NASA's STARC_ABL aircraft design is made through a CFD based design approach. This method employs three iterative steps to exploit the CFD tools until the design requirements are met: a quasi-2D through-flow model to design the fan/EGV, a 3-D RANS simulation of the single blade row to account for the inlet/fan and the EGV/nozzle interaction, and a 3-D RANS simulation of the airframe with a propulsor installed - propulsion airframe integration (PAI). The design requirements which include the thrust, and shaft power of the propulsor are matched throughout the evaluations coming from two CFD domains, i.e., the turbo-machinery and the PAI. During the switch between these different computational domains, the inlet and exit profiles are matched via the correction factors of the body-force model.

The present tail-cone thruster (TCT) aerodynamic design leverages a low-pressure ratio fan (FPR=1.2 1.25) of which the camber-line angles are predicted by a quasi-2D through-flow model. The quasi-2D model is derived to analyze the radially distorted flow resulting from the ingested boundary layer at the inlet. It also estimates the appropriate velocity vectors of the metal angles of the fan and EGV which is subjected to different types of vortex at the fan exit.

The baseline geometry is revisited and its internal flow-path and exhaust cone are redesigned to illustrate the strong correlation among the components of the propulsor in the PAI domain. The peak efficiency point of the fan/EGV with respect to the blade counts, a.k.a. solidity, and rotational speed is chosen
\end{abstract}

for the cruise condition via parametric studies. The corresponding performance maps are presented. The resulting performance metrics of the new conceptual design of the BLI propulsor are analyzed and compared with these of the baseline in the PAI aspect. Finally, ideas of the CFD based design of a BLI propulsor are discussed based on the observations drawn from the numerical results.

\section{INTRODUCTION}

Over the last few decades, extensive efforts have been made to understand the benefits of the boundary layer ingestion (BLI) since it was introduced to the field of the aviation propulsion. ${ }^{1}$ The efforts include the conceptual designs of whole propulsion systems ${ }^{2}$, aerodynamic designs of inlet ${ }^{3}$, propulsor $^{4}$, aeromechanics studies of the distortion-tolerant fan ${ }^{5}$, and many other remarkable studies. Recently, NASA and UTRC conducted an inlet-propulsor test and presented several promising results ${ }^{6}$. The results showed not only benefits in the fuel burn reduction, but also high stall margin and aeromechanical robustness. In addition to the test rig based studies, various aircraft concepts including the tail-cone thruster (STARC-ABL) ${ }^{7}$, double-bubble $(\mathrm{D} 8)^{8-9}$, and hybrid wing-body $(\mathrm{N} 3-\mathrm{X})^{10}$ aircrafts have been invented and designed to take advantage of the BLI benefits. The BLI propulsion system is strongly coupled with the airframe, thus, it has been difficult to separate the thruster from the other components, i.e., airframe, inlet, and nozzle ${ }^{11}$ even at the conceptual design phase.

Recently, Welstead et al. ${ }^{7}$ carried out a system design of STARC_ABL which is one of NASA's recent concepts of the next generation aircraft. In this concept, a nacelle is installed at the tail-cone and ingests the wake from the fuselage. Therefore, 
the incoming boundary layer into the fan face is concentric, and the inlet distortion thus is more axi-symmetric relative to other embedded BLI engine types. The circumferential distortion at the cruise condition is predicted to be less than $3 \%$ even with a vertical stabilizer after a shape optimization. ${ }^{22}$ The benefit is estimated to achieve about a $12 \%$ fuel burn saving. The design of the baseline aircraft resulting from the system study is summarized in Table1, including the design, mission, and nacelle specifications. However, the 1-D engine model adopted in the system design process showed limitations to incorporate the physical complexity of the BLI, thus, the resulted geometry is not favorable for healthy fan operation. The proposed internal flow-path was not appropriate for the flow contraction through the fan and EGV stage. Thus, further investigations and improvements of each component with higher fidelity models are needed.

In the present study, a design of the propulsor system is carried out to refine the baseline design into a more realizable shape. The baseline configuration is modeled in three different fidelities: a quasi-2D through-flow model; a 3-D Reynolds Averaged NavierStokes (RANS) model coupled with body force as source terms in the flow equations for PAI; and a 3-D RANS turbo-machinery CFD model. The profiles from the two low-fidelity models in terms of the turbo-machinery simulation are validated by the numerical results from the 3-D RANS turbo-machinery CFD model. The design efforts of the propulsor system including parametric studies of the sizing of the nacelle, the fan/EGV parameters such as the pressure ratio, rotational speed, solidity, as well as the redesign of the internal flow path, and the exhaust cone are made to realize the benefit of the aircraft system. Hereafter, the performance metrics that are specific for the boundary layer ingestion engines will be addressed and followed by the detailed introductions of the numerical technics. The results and discussions will be presented in the order as follows; (I) the investigation of the effect of the boundary conditions and the feasibility of the assumption for a de-coupled analysis and design of the propulsor, (II) validations of the body-force and the quasi-2D through-flow models, (III) designs of the internal flowpath and nozzle/exhaust cone and (IV) fan design (V) fan performance at the operating conditions (VI) PAI performance. Finally, the performance of the designed configuration is evaluated and compared with the baseline geometry.

\section{BENEFITS OF BOUNDARY LAYER INGESTION}

The performance benefit of the BLI stems from the low momentum boundary layer flow entering the inlet. Consequently, the required power is lower than a thrust equivalent propulsor with a clean flow inlet, i.e., the non-BLI propulsor. To gauge the benefit of the BLI, two major performance metrics suggested by Smith $^{1}$ are the power saving coefficient (i.e. the reduction of power for the same thrust) and propulsive efficiency $\left(\eta_{p}\right)$. Smith also named the form (shape) factor of the incoming boundary layer, and the wake recovery factor as the main critical factors to maximize the power saving and the propulsive efficiency. Here, the form factor $(H)$, the ratio of the wake momentum area $(\theta)$ to the displacement area $(\delta)$, is used to describe the low momentum flow into the propulsor. In Ref. 1 , he proved that a higher form factor can be redeemed by a higher power saving due to smaller ram drag. From the airframe design perspective, however, excessively high form factor may cause flow separation which will increase the drag and lower the total pressure recovery of the inlet. Thus, Hall et al. ${ }^{9}$ derived a power balance method to compromise the benefits of the drag reduction and the power saving of the propulsion system simultaneously. In the present BLI study, the authors recognize that the installation of a fan will not affect the potential flow field of the fuselage as long as no significant flow blockage is caused by the fan operation (i.e. throughout the fan operating range) and the incoming boundary layer profile does not incur any separations. For a ducted propulsor, however, the flow blockage of the nacelle does affect the upstream flow. Thus, the airframe, inlet and nacelle can be decoupled from the rest of the propulsor components design. This assumption can be verified by comparison of the upstream flow field for different mass flow rates in the figure 1 . Here, the station 1 is located upstream from the nacelle, 2 for right at the inlet and 3 for at the AIP.

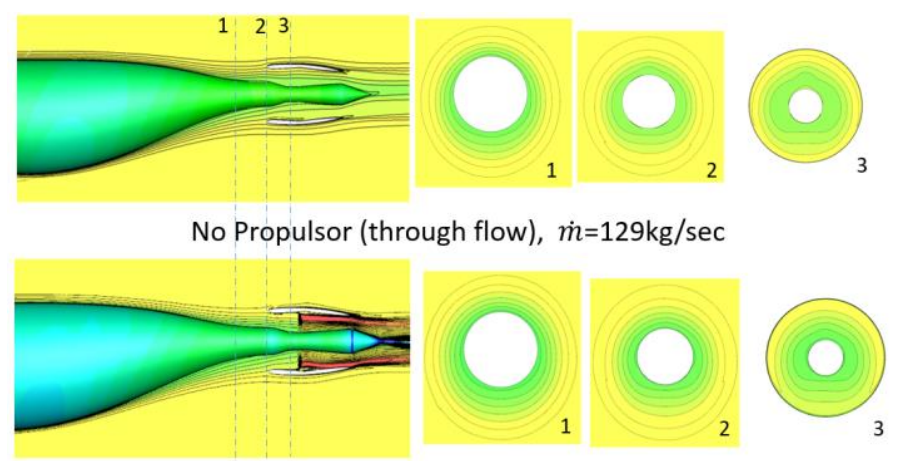

Fan Modeling (GE-R4), $\dot{m}=163.1 \mathrm{~kg} / \mathrm{sec}, \mathrm{T}=14.6 \mathrm{kN}$ (3210 lbf)

Figure 1. The effect toward the upstream flow pattern from the fan operation is shown by comparing the total pressure contours at selected upstream stations from the fan face. The case with body force model is on the bottom and the top figure is for the case without body force.

Figure 2 displays the control volume of interest in the present study superposed on top of the total pressure contours which is analyzed by a 3-D RANS solver. The performance metrics are evaluated based on the control volume defined in the figure. The notations of the stations are provided in the nomenclature. Another important performance metrics of the BLI propulsor introduced in the Ref. [1] is the propulsive power $\left(P_{p}\right)$ which measures the mechanical power generated by the propulsor as given in Eq. (1).

$$
P_{p}=\int_{A_{e}} \rho V_{z}\left(\frac{1}{2}|\vec{V}|^{2}+\frac{p_{s}}{\rho}\right) d A_{z}-\int_{A_{i}} \rho V_{z}\left(\frac{1}{2}|\vec{V}|^{2}+\frac{p_{s}}{\rho}\right) d A_{z},
$$

where $A_{e}$ and $A_{i}$ are area of the exit and inlet respectively, see Fig. 2. And, $\vec{V}$ is the velocity vector of $\left(V_{z}, V_{r}, V_{\theta}\right) ; p_{s}$ is the static pressure and $A_{z}$ is the projected area in the axial z direction. Thus, adopting the definition of the propulsive power, the propulsive efficiency, $\eta_{p}$ can be evaluated by Eq. (2), 


$$
\eta_{p}=V_{\infty} T / P_{p}
$$

where $T$ is the net thrust as given in Eq. (3) and $V_{\infty}$ denotes the free-stream velocity of the aircraft.

$$
T=\int_{A_{e}}\left(\rho V_{z}|\vec{V}|+p_{s}-p 0_{r}\right) d A_{z}-\int_{A_{i}}\left(\rho V_{z}|\vec{V}|+p_{s}-p 0_{r}\right) d A_{z},
$$

where $p 0_{r}$ is the ambient pressure.

Since the propulsive power does not include the thermal power generation which is reflecting the loss throughout the propulsor, the actual shaft power is defined as in Eq. (4)

$$
P=\int_{A_{e}} \rho V_{z}\left(\frac{1}{2}|\vec{V}|^{2}+\frac{p_{s}}{\rho}+C_{p} T_{s}\right) d A_{z}-\int_{A_{i}} \rho V_{z}\left(\frac{1}{2}|\vec{V}|^{2}+\frac{p_{s}}{\rho}+C_{p} T_{s}\right) d A_{z}
$$

By introducing the ratio of the propulsive power to the actual shaft power which is denoted by $\eta_{K E}$, the overall propulsor efficiency, $\eta$, can be evaluated via Eq. (5).

$$
\eta=\eta_{p} \eta_{K E}=V_{\infty} T / P
$$

\begin{tabular}{|c|c|c|c|c|c|c|c|}
\hline \multirow{2}{*}{$\begin{array}{c}\text { Geometric } \\
\text { Spec. }\end{array}$} & $\mathbf{A}_{\mathbf{c}}\left(\mathbf{m}^{2}\right)$ & $\mathbf{A}_{\mathbf{i}}\left(\mathbf{m}^{2}\right)$ & $\mathbf{A}_{\mathbf{e}}\left(\mathbf{m}^{2}\right)$ & $\mathbf{H}_{\mathbf{i}}(\mathbf{m})$ & $\boldsymbol{\Phi}\left({ }^{\circ}\right)$ & $\mathbf{D}_{\mathbf{f}}(\mathbf{m})$ & $\mathbf{L}(\mathbf{m})$ \\
\cline { 2 - 8 } & 2.47 & 2.08 & 1.73 & 0.54 & 20 & 1.83 & 38 \\
\hline \multirow{3}{*}{$\begin{array}{c}\text { Operating } \\
\text { Conditions }\end{array}$} & $\mathbf{P}(\mathbf{k W})$ & $\mathbf{T}(\mathbf{k N})$ & $\mathbf{F P R}$ & \multicolumn{2}{|c|}{$\mathbf{N}(\mathbf{r p m})$} & $\dot{\mathbf{m}}(\mathbf{k g} / \mathbf{s e c})$ \\
\cline { 2 - 8 } & 2610 & 14.7 & 1.25 & \multicolumn{2}{c|}{2500} & 157.08 \\
\cline { 2 - 8 } & \multicolumn{2}{|c|}{$\mathbf{M}_{\infty}$} & \multicolumn{2}{|c|}{$\mathbf{R e} / \mathbf{m}$} & Alt.(ft.) & $\mathbf{A O A}\left({ }^{\circ}\right)$ \\
\cline { 2 - 7 } & \multicolumn{2}{|c|}{0.785} & $5.67 \mathrm{e}+06$ & \multicolumn{2}{c|}{37,000} & \multicolumn{2}{c|}{2.0} \\
\hline
\end{tabular}

Table 1. Specification of the airframe/nacelle and operating conditions in the cruise flight. ${ }^{7}$

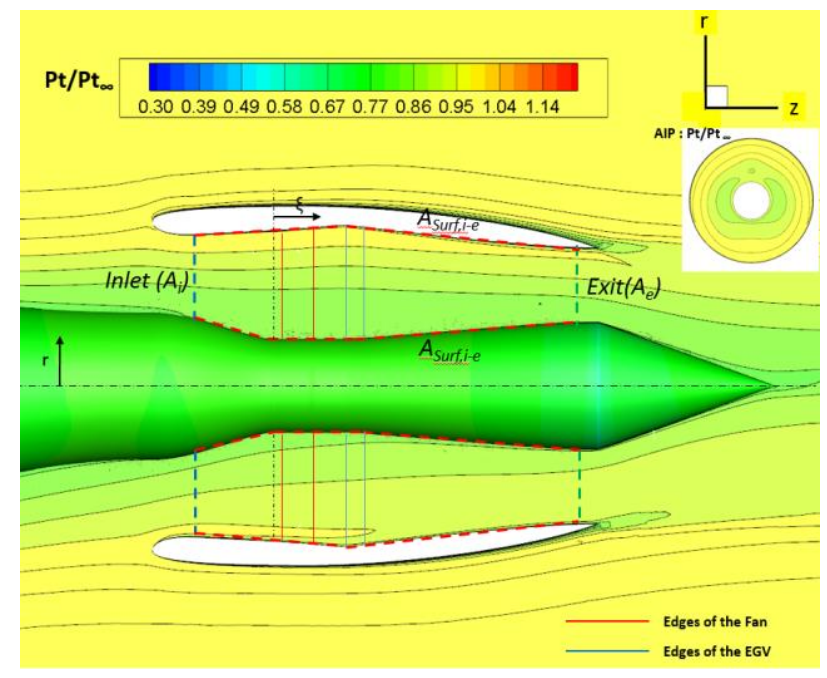

Figure 2. Control volume for the performance measurement of the BLI propulsor. The total pressure contours of the tail cone thruster baseline design ${ }^{7}$ are from a 3-D RANS CFD. The inserted plot shows the circumferential total pressure contours at the AIP. Also, the location of the fan and EGV interface planes in the turbo-machinery domain are defined here.

\section{MULTI-FIDELITY DESIGN \& ANALYSIS FRAMEWORK}

The conceptual design framework is composed of three numerical models and two computational domains. Those domains and numerical models will be described in the following. A flowchart is drawn at the end of this section to show the steps and connections among all the modules in the framework.

As aforementioned, the circumferential distortion at three different operating conditions (different angles of attack) are lower than 3\% in the SAE standard DPCP as described in the Ref. 22. Therefore, the present conceptual design study is focused on the axi-symmetric radial distortion at the fan face. Thus, it is noted that the models used for the turbo-machinery analyses are adopting the axi-symmetric methods, such as a single passage time-steady CFD, the mixing plane, and a 2-D through-flow model. The numerical results from the turbomachinery models are cross-checked with a full 3-D RANS model in the PAI modeling.

Domains: Two sets of the computational domains are used, one for the detailed turbo-machinery analysis and the other for the PAI analysis. The aerodynamic interface plane (AIP) shown in Fig. 2 is located at $z=35 \mathrm{~m}$ which is based on the airframe coordinate starting from the nose of the fuselage and is used as the origin in the engine coordinate. The symbol $\xi$ denotes the relative distance measured from the AIP, a negative $\xi$ indicating upstream and positive for downstream from the AIP. The dashed lines depict the inlet and the exit boundaries of the domain of the turbo-machinery analyses. The red solid lines indicate the boundaries of the fan and blue solid ones for the EGV. Both the quasi-2D and fan/EGV CFD models get the inlet profiles (at $\xi=-$ $0.367 \mathrm{~m}$ ) as input and the exit domain is located at $\xi=1.753 \mathrm{~m}$.

The fan face is located at $\xi=0.076 \mathrm{~m}$ ( $3 \mathrm{in}$.) and fan trailing edge is at $\xi=0.279 \mathrm{~m}$ ( $11 \mathrm{in}$.) following the engine coordinate. The baseline geometry has $1.890 \mathrm{~m}$ (74.4 in.) fan diameter and the hub to tip radius ratio of the fan is 0.3 . The location of the leading edge of the $\mathrm{EGV}$ in the meridional plane is at $\xi=0.443 \mathrm{~m}$ (17.42 in.) and the trailing edge is at $\xi=0.583 \mathrm{~m}$ (22.96 in.).
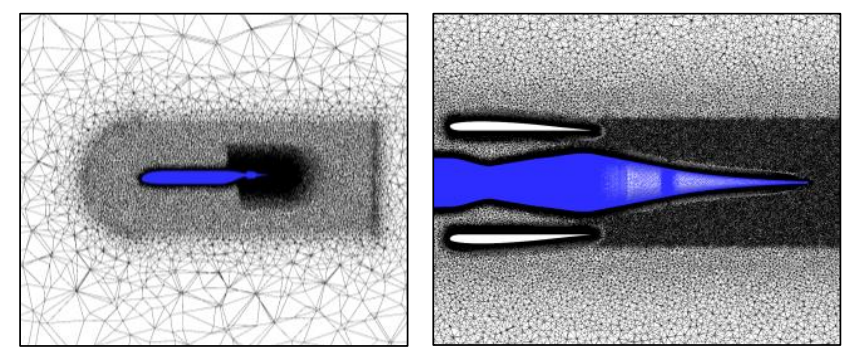

Figure 3. Meshes for PAI modeling.

Propulsion-Airframe Integration with body force model: To account for the flow turning, pressure rise and loss effects of the fan blades and the inlet-fan interactions in the integrated airframe-propulsion applications, this module is to provide a more effective modeling than the direct coupling of the airframe, inlet and full-annulus fan blades in the computational domain. A full-scale fuselage geometry with the tail-cone nacelle is modeled via an unstructured RANS mesh in a free-stream condition in order to incorporate the interaction of the fan operation and the upstream boundary layer ingestion as well as the downstream jet flow of the nozzle. The specification of the baseline fuselage, nacelle geometries are listed in Table 1. The 
CFD code used for the PAI model is Go-flow which is a 3-D unstructured RANS solver in Cartesian coordinate system. ${ }^{10,11,21}$ Both the internal and external flow fields are assumed to be fully turbulent since most of the nacelle domain is embedded in the boundary layer of the fuselage. The Spallart-Almaras turbulence model is adopted in the calculations. The size of total meshes reaches about 18 million cells, based on the number of the mesh vertices as shown in Fig. 3. It is noted that the flow-path and the downstream meshes are kept fine enough to resolve the wake profile.

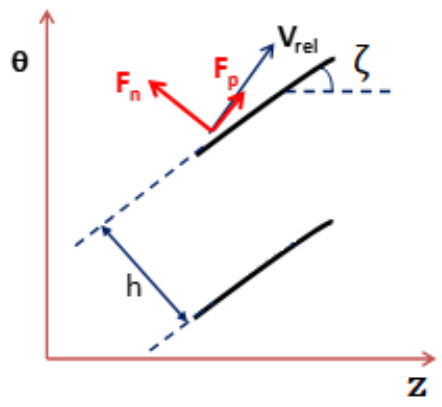

Figure 4. Force decomposition of the body-force.

Body-Force Model: Instead of a computationally intensive full-annulus simulation, we adopt the body-force model developed by Kim et al. ${ }^{21}$ to model the flow turning and loss throughout the rotor/stator blade rows subjected to the distorted inflow conditions. The formulation of the body force model ${ }^{21}$ is based on the normal and parallel force components driven by the turning from the camber angles $(\zeta)$ and the rotation of the blades as shown in Fig. 4. Assuming an infinite number of the blades and axisymmetric flow in each infinitesimal blade passage, the cascade blade forces can be modeled by a pair of the normal and parallel force components. The normal force component is calculated by equation (6).

$$
F_{n}=\frac{K_{n}}{h} V_{n} V_{p}+\frac{2}{c} \sin \left(\frac{\Delta \zeta}{2}\right) V_{n}^{2}
$$

where $K_{\mathrm{n}}$ is the normal force coefficient. As shown in Fig. $4, h$ is the blade-to-blade gap-staggered spacing given by

$$
h=\frac{2 \pi r \sqrt{\sigma} \cos \zeta}{N B}
$$

where $r$ is the radius, $\sigma$ is the solidity, $\zeta$ is the local blade camber angle, and $N B$ is the number of blades. In Eq. (6), $V_{n}$ and $V_{p}$ are the velocity components normal and parallel to the local cascade flow and are defined as follows:

$$
\begin{aligned}
& V_{n}=V_{\theta} \cos \zeta-V_{z} \sin \zeta \\
& V_{p}=V_{\mathrm{z}} \cos \zeta-V_{\theta} \sin \zeta
\end{aligned}
$$

where $V_{z}$ and $V_{\theta}$ are the velocity components in the axial and circumferential directions. In the second term on the right-hand side of Eq. (6), $c$ is the chord length and $\Delta \zeta$ is the camber angle difference between the trailing edge $(T E)$ and leading edge $(L E)$ :

$$
\Delta \zeta=\zeta_{T E}-\zeta_{L E}
$$

The axial and circumferential components of the normal force are calculated by

$$
F_{n, z}=F_{n} \frac{V_{\theta}}{V_{\text {rel }}},
$$

and

$$
F_{n, \theta}=F_{n} \frac{V_{Z}}{V_{\text {rel }}},
$$

where $V_{\text {rel }}$ is the magnitude of the blade relative velocity.

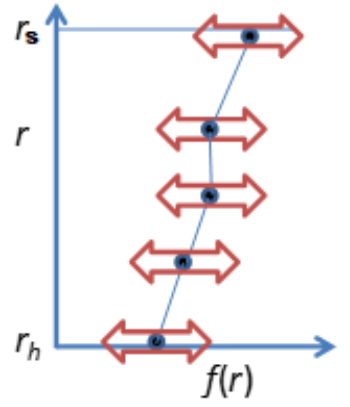

Figure 5. Definition of $f(r)$ for the body force coefficient $K_{n}$.

Defoe $^{23}$ suggested an empirical model to determine the normal force coefficient $K_{n}$ for a particular fan rotor:

$$
K_{n}=(4.2-3.3 \zeta)\left[4.172\left(\frac{r-r_{h}}{r_{s}-r_{h}}\right)^{2}-3.118\left(\frac{r-r_{h}}{r_{s}-r_{h}}\right)+2.145\right] \text {, (13) }
$$

where $r_{h}$ and $r_{s}$ are the hub and tip radius of the fan blade respectively. The first expression in parentheses in Eq. (13), an empirical term suggested by $\mathrm{Gong}^{20}$, is multiplied by the bracketed expression to adjust the magnitude of $K_{n}$ along the spanwise direction. Kim et al. ${ }^{21}$ extended the formulation of $K_{n}$ in Eq. (13) by introducing a general function $f(r)$ to allow turning adjustment:

$$
K_{n}=(4.2-3.3 \zeta) f(r)
$$

where $f(r)$ is a set of line segments connecting control points distributed along the blade span as shown in Figure 5. The control points of $f(r)$ were adjusted to incorporate the turning defect by matching the profiles with a turbo-machinery CFD modeling in the present study.

Marble $^{24}$ suggested a loss force model relating the entropy production and the parallel body force as follows:

$$
F_{p}=-T \frac{V_{m}}{V_{r e l}} \frac{\partial s}{\partial m}
$$

where $T$ is the temperature, $s$ is the entropy, and $m$ is the coordinate along the meridional streamline and $V_{m}$ is the meridional velocity component. Here, we made an assumption that the entropy production is constant along the blade span and chord for a simplicity. The spanwise variation of the entropy production, however, can be readily adopted in the body force modeling process when the required data for the modeling are available. With the assumption, we can calculate the averaged entropy change, either from test data or CFD results for the total pressure, $P_{t}$, and total temperature, $T_{t}$, ratios across a blade row, by using the canonical equation of state: 


$$
\frac{\Delta s}{\underline{\mathrm{R}}}=\frac{\gamma}{\gamma-1} \ln \left(\frac{T_{t, 2}}{T_{t, 1}}\right)-\ln \left(\frac{P_{t, 2}}{P_{t, 1}}\right)
$$

where $\underline{\mathrm{R}}$ is the gas constant, $\gamma$ is the specific heat ratio, and stations 1 and 2 refer to the entrance and exit of a fan blade row, respectively.

QUASI-2D Through-Flow Model: As mentioned above, there are three different BLI configurations currently being investigated under NASA Advanced Air Transportation Technologies BLI electric propulsion projects: tail-cone thruster (STARC-ABL) ${ }^{7}$, double-bubble (D8) ${ }^{8-9}$, and hybrid wing-body $(\mathrm{N} 3-\mathrm{X})^{10,11}$. These concepts can be classified by the shape of distortion at the fan face. The embedded configurations, such as the N3-X and D8, have significant circumferential distortion ingested into the inlet as shown in Figure 6 while the tail-cone thruster has the inlet flow closer to the axisymmetric distortion ${ }^{16}$. Both types of the distortions affect the fan operability and performance critically. The axisymmetric boundary layer ingestion has a radially dominant distortion as shown in Figure 2 while the embedded BLI system exhibits both circumferential and radial distortion as shown in Figure 6.

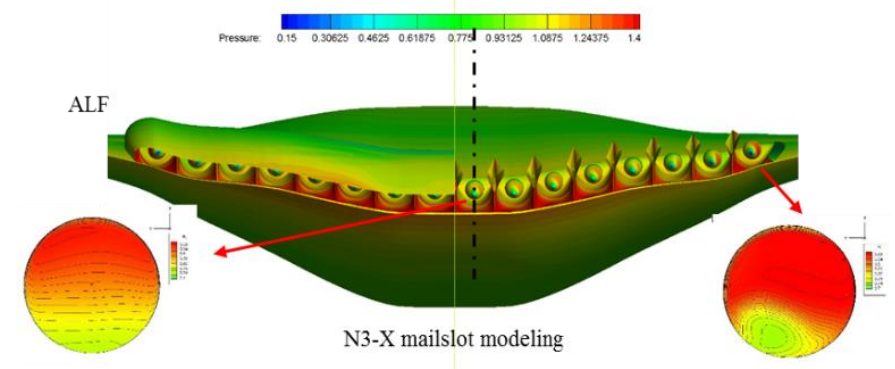

Figure 6. RANS CFD with the body-force model of the N3-X mailslot propulsors ${ }^{21}$. Shown on the left is the total pressure contours of the first propulsor located from the center body symmetry plane as indicated by the black dotted line; on the right is the total pressure contours of the $8^{\text {th }}$ propulsor.

As the incoming flow to the tail-cone thruster is relatively axisymmetric, the present design work leverages 2-D throughflow model for a short design cycle. However, in dealing with boundary layer flows, the stream-line (SL) curvature model sometimes suffers from convergence issue during the reconstruction process of the stream lines in case of the existence of the endwall defects. Thus, it has been difficult to utilize the SL model if the endwall profile is significantly weak. Consequently, a new and robust quasi-2D through-flow method which replaces the iterative stream-line calculation with the redistribution of the stream-tube area along the quasi-normal is suggested. It is devised to perform the velocity vector study of a given internal flow-path, meridional projections of blades and vanes, and radially distorted inlet profiles from CFD. The key features of the model are (a) the definition of the quasi-normal along the blade edge projection; (b) the work profile design; (c) a sequential solution procedure along each quasi-normal to avoid numerical instability from the iterative matrix solver; and (d) the radial momentum equilibrium equation in a conservative form.
As an example of the current modeling, the stations of the quasi-normal in the flow-path of the GE-R4 fan/EGV system are presented in Figs. 7-(a). ${ }^{17}$ The streamlines and the flow-path are defined in a discrete manner on each station. Figure 7-(b) shows a streamline definition through the blade edge projection. Physically, the meridional streamline will locally pass the blade edge in the perpendicular direction. Thus, in order to get rid of a source of numerical error in angle definition, $\varepsilon$, the meridional angle, $\varphi$, and quasi-normal angle, $\lambda$, are equated as in Eq. (17).

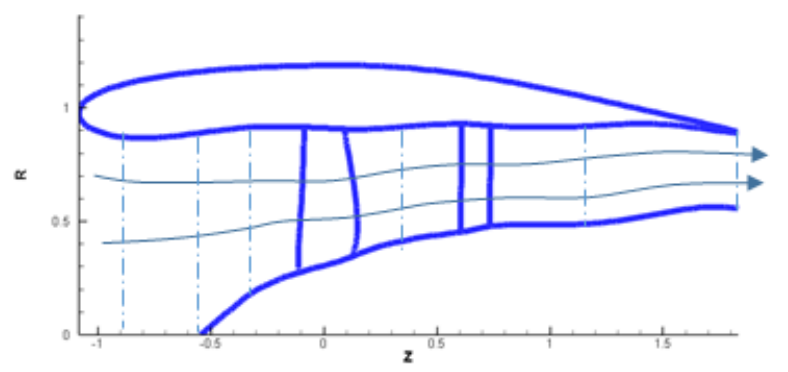

(a) Definition of quasi-normal stations in meridional view where the axis $\mathrm{R}$ is the radius of the cone in a schematic GE-R4 fan-EGV system (cylindrical coordinate).

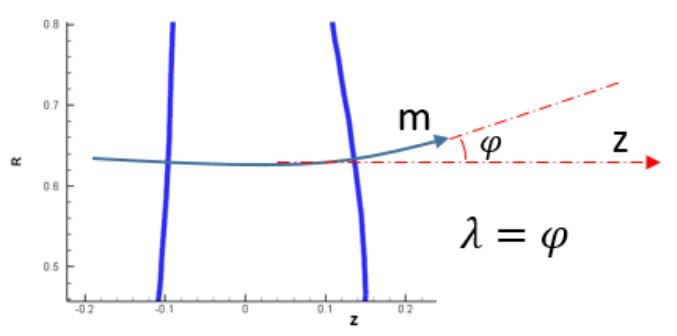

(b) The meridional direction of streamline through edge stations.

Figure 7. Streamline and quasi-normal definition.

As a result, the differential operator along quasi-normal $(y)$ and that along normal direction $(n)$ relative to meridional direction will be equated as given in Eq. (18).

$$
\begin{gathered}
\varepsilon=\phi-\lambda \sim 0 \\
\frac{\partial}{\partial n}=\frac{1}{\cos \varepsilon}\left[\frac{\partial}{\partial y}-\sin \varepsilon \frac{\partial}{\partial m}\right]=\frac{\partial}{\partial y},
\end{gathered}
$$

Here, $n$ is the coordinate along the normal direction and $m$ is meridional coordinate. Equation (19) represents the conventional momentum equation in a conservative form as below;

$$
\frac{\partial \vec{M}}{\partial \mathrm{t}}-\vec{V} \times(\vec{\nabla} \times \vec{M}+2 \rho \vec{\omega})=\rho T \vec{\nabla} s-\rho \vec{\nabla} I
$$

$\vec{M}$ depicts the momentum vector, which is the product of the local density and relative velocity vector, $\vec{W}$. Each component of the momentum vector can be represented by $\left(M_{m}=\right.$ $\left.\rho W_{m}, M_{\theta}=\rho W_{\theta}, M_{n}=\rho W_{n}\right)$ in the meridional, tangential and normal directions, respectively. With the above assumptions in Eqs. (17) and (18), the radial component of Eq.(19) can be rewritten as Eq.(20) with time-steady, and axi-symmetric assumptions. 


$$
\begin{gathered}
\frac{\partial M_{m}}{\partial \mathrm{y}}+\left(k_{m} \cos ^{2} \beta+\frac{\sin \beta-\cos \beta}{r} \frac{\partial(r \tan \beta)}{\partial \mathrm{y}}\right) M_{m}+2 \rho \omega \cos \beta \sin \beta \cos \varphi \\
=\frac{\rho^{2} \cos ^{2} \beta}{M_{m}}\left(\frac{\partial I}{\partial y}-T \frac{\partial s}{\partial y}\right),
\end{gathered}
$$

where $I$ is the rothalpy, $t$ is time, $s$ is the entropy, $\beta$ is relative flow angle against the meridional direction and $k_{m}$ is the meridional derivative of $\varphi$, i.e., $k_{m}=\frac{\partial \varphi}{\partial m}$, and $\varphi$ is the flow angle between meridional direction and axial direction. The derivation process of Eqs. (19) and (20) in the conservative variable form follows the Aungier's derivation of the primitive variable form ${ }^{13}$ and the details are given in Ref. 13. It is noted that the $\partial V_{m} / \partial m$ term which appears in the conventional momentum equation and needs iterative calculation between neighboring stations is eliminated. Furthermore, solving the conservative form of the equation (20) provides a simple control of the area of each stream tube as shown in Eq.(21) as each stream tube has constant mass flow rate. The area re-distribution process replaces the traditional re-construction process of the streamlines.

$$
M_{m}=\rho W_{m}=\frac{\Delta m_{l} t h}{\Delta A_{i} t h}, \text { thus, } \Delta A_{i} t h=\frac{\Delta m_{l} t h}{M_{m}},
$$

where $m_{l^{t h}}$ is the mass flow rate at the $i^{\text {th }}$ stream tube and $A_{i^{t h}}$ here is the area of the $i^{\text {th }}$ stream tube.

Moreover, the solution of equation (20) can be obtained by using a solution of the non-linear ordinary differential equation with a simple constraint of quasi-normal area for the ducted flows. ${ }^{13}$

Work Profile Design: To design a rotor, the designer can choose the radial turning distribution depending on the stage reaction, fan pressure ratio and the characteristics of the incoming flow profiles. The radial work profiles per unit mass flow by a rotor can be given as in equation (22).

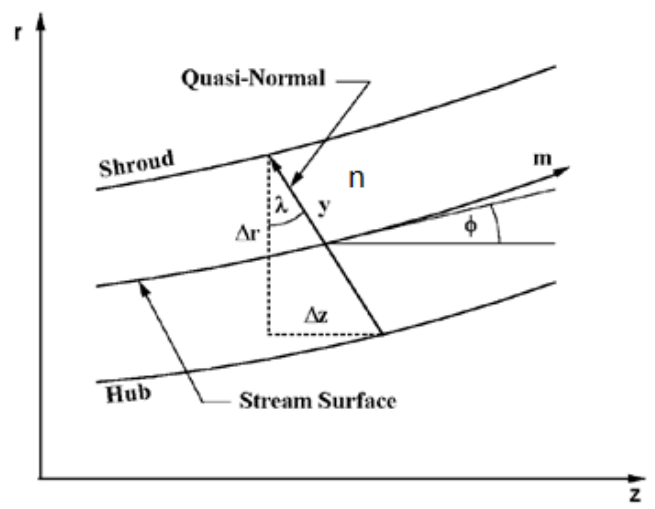

Figure 8. Quasi-normal angle and meridional angle definition at quasi-normal stations. [Ref. 13]

$$
\mathbb{W}=C_{1}+C_{2} r^{l}
$$

The equation (22) represents a general form of the work profiles, i.e., free vortex if $l=0$ and forced vortex type in case of $l>0$. To showcase the above described models of the quasi-2D and work profile, GE-R4 is used for validation purpose. A forced vortex work profile of $l=3$ case is derived and later compared with the CFD computations of the GE-R4 fan exit profile at a part speed $\left(85 \% N_{c}\right.$, subsonic).

For a clean inlet flow, the drag force of a blade in high-speed is in proportion to the square of the tangential velocity of the blade. $^{25}$ The required work to overcome the drag force of the blade rotation can be represented as in Eq. (23)

$$
\mathbb{W}_{\text {loss }}=\frac{F_{\text {drag } U}}{\dot{m}}=\frac{1}{2} \frac{\rho U^{2} C_{d} U A_{m}}{\rho W_{m} A_{\theta}} \sim C_{d} r^{3}
$$

$F_{\text {drag }}$ denotes the local drag force, $\dot{m}$ is the mass flow, $U(=r \omega)$ is the tangential velocity of the blade, and $C_{d}$ is the drag coefficient of the blade geometry. $A_{m}$ and $A_{\theta}$ are the projected area on the meridional and tangential directions. The required shaft work is a sum of the ideal free-vortex work and the additional work for the drag. Thus, it is expressed as a function of radius as in Eq.(24), see Figure 9.

$$
\mathbb{W}_{\text {req }}=\mathbb{W}_{\text {ideal }}+\mathbb{W}_{\text {loss }}=C_{1}+C_{2} C_{d} r^{3},
$$

where the drag coefficient is radially constant and proportional to the $C_{l}{ }^{2}$. It can be found that $C_{d} \sim 0.18 C_{l}^{2}$ and $C_{l}^{2} \sim\left(r V_{\theta}\right)^{2}$ in Ch.6, Ref.13.

If we adopt a target polytropic efficiency in the radial function of the work equation (24),

$$
\mathbb{W}_{\text {req }}(r)=C_{p} T_{t, 1}\left(\left(P R_{\text {des }}\right)^{\frac{\gamma-1}{\gamma}}-1\right)+C_{2}^{\prime} r^{3}
$$

where $C_{2}^{\prime}=\frac{5}{2} C_{p} T_{t, 1} \frac{r_{s}^{2}-r_{h}^{2}}{r_{s}^{5}-r_{h}^{5}}\left(\left(P R_{\text {des }}\right)^{\frac{\gamma-1}{\gamma}}-\left(P R_{\text {ideal }}\right)^{\frac{\gamma-1}{\gamma}}\right)$ based on the mean values at the center of the exit area.

and the design pressure ratio will be

$$
P R_{\text {des }}=\left(T_{t, 2} / T_{t, 1}\right)^{\frac{\gamma}{\gamma-1} \eta_{p}}
$$

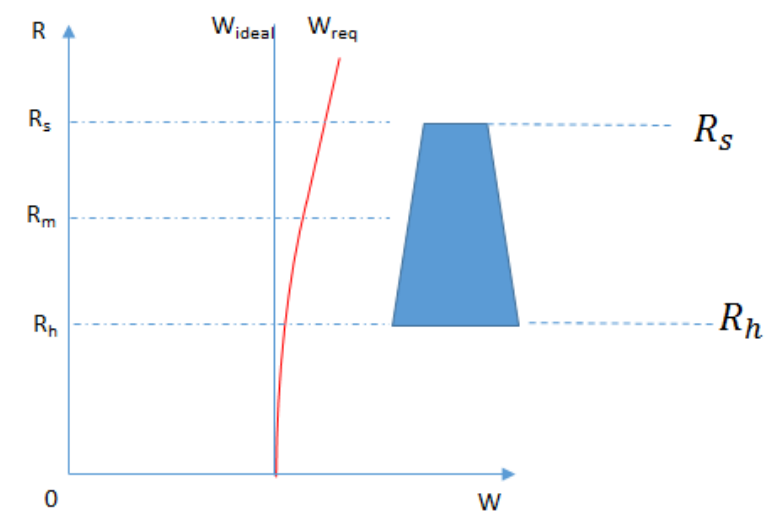

Figure 9. Required work profile and ideal free vortex work ( $r_{\mathrm{s}}$ and $\mathrm{R}_{\mathrm{s}}$ : tip radius, $\mathrm{r}_{\mathrm{h}}$ and $\mathrm{R}_{\mathrm{h}}$ : hub radius)

The function of the radial work profile is imposed in the rothalpy calculation during the quasi-2D model for the GE-R4 fan. The turning angle predicted by quasi-2D model with the design work profile is compared with RANS CFD ${ }^{15}$ result at $85 \% N_{c}$ in Figs. 10 and 11 . The turning prediction looks 
reasonably close to the CFD profile as shown in the figures, and the total pressure profile were observed to be in a good agreement with CFD profiles in Fig. 11.

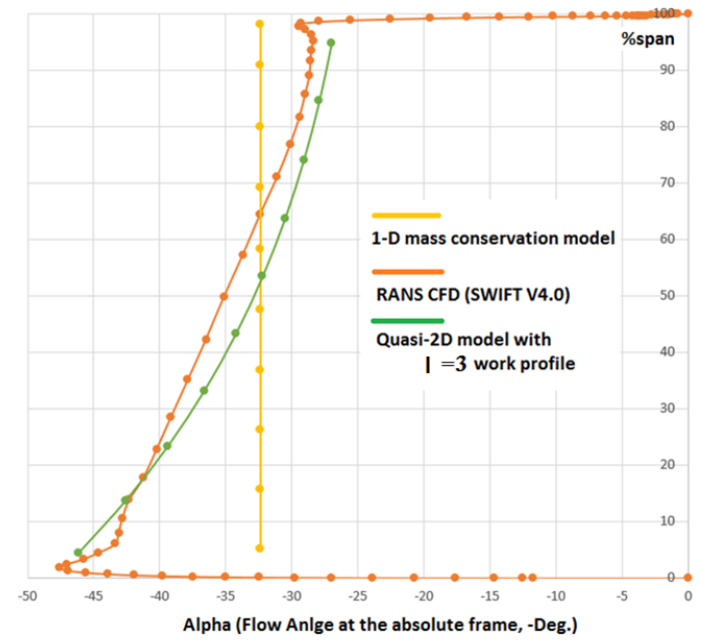

Figure 10. GE-R4 fan trailing edge, Turning prediction $(-\alpha$, Deg.), at $85 \% \mathrm{~N}_{\mathrm{c}}$ and $\dot{\mathrm{m}}_{\mathrm{c}}=93.6 \mathrm{lbm} / \mathrm{s}$.

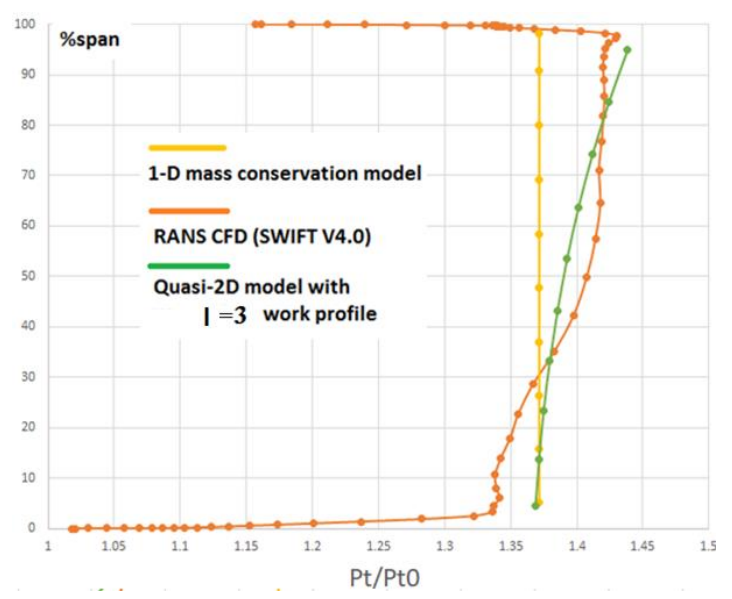

Figure 11. GE-R4 fan trailing edge, non-dimensional total pressure, at $85 \% \mathrm{~N}_{\mathrm{c}}$ and $\dot{\mathrm{m}}_{\mathrm{c}}=93.6 \mathrm{lbm} / \mathrm{s}$.

Regarding loss models, Lieblein's design angle of attack (incidence), design deviation angle models ${ }^{18}$ are used for obtaining metal angles out of the quasi-2D model. End-wall loss model is not applied in the model for now since the inlet profile already has massive low momentum flow from the boundary layer ingestion and the entrainment boundary layer well preserved through rotor stage to the EGV in a single stage model. Since the model assumed the quasi-normal over a blade only at the edges, the meanline angle distribution is assumed to be a circular arc airfoil during the conceptual design. Thickness distribution is following NACA65 series airfoil to leverage Lieblein's models. The maximum thicknesses of the fan at the tip and hub are $2 \%$ and $7.5 \%$ for the metal chord at the respective stream lines. The maximum thickness of the EGV is about $6 \%$ per the chord length at the pitch-line. A simple chocking condition $\left(M^{2}<1.1\right)$ is applied to prevent choking through the flow-path.

Fan/EGV CFD modeling: This module is to perform turbomachinery flow simulations in order to provide the body force coefficients for Goflow code and validate the radial profiles. The designed fan and EGV geometries together with the tailored flow-path are gridded by TCGRID-V4.0 which is a multi-block mesh generator of turbo-machinery blades. As shown in Figs. 12, the inlet domain is H-type mesh in the stationary domain and interfaced with $\mathrm{C}$-type blade mesh domain in the rotational frame with a mixing plane. The downstream EGV is gridded in C-type mesh and the interface between rotor and stator is also modeled via mixing plane. The CFD solver used is the SWIFT V.4.0, which is a 3-D RANS turbomachinery blade rows analysis code, adopting multi-block, periodic condition for blade to blade, and utilizing mixing plane between the blade rows. The AUSM + is used for the flux-function of the convection terms with the $2^{\text {nd }}$ order spatial accuracy, the viscous terms are using $2^{\text {nd }}$ order central difference. The time integration scheme is multi-stage Runge-Kutta scheme. The Wilcox's $2006 k-\omega$ model with a fully turbulent flow assumption is applied for the turbulence modeling. The turbulent stresses are limited by a stress limiter. The mesh size for the rotor and stator are $(265 \times 43 \times 83)$ in the streamline, blade to blade and span-wise direction, respectively. The $\mathrm{y}^{+}$is maintained lower than 5 over the blade and vane. The effect of the rotor tip clearance is ignored during the conceptual design. OpenMP is used for a multi-core parallel processing.

Flowchart: The developed multi-fidelity design and analysis framework is summarized in flowchart and in descriptions as follows.

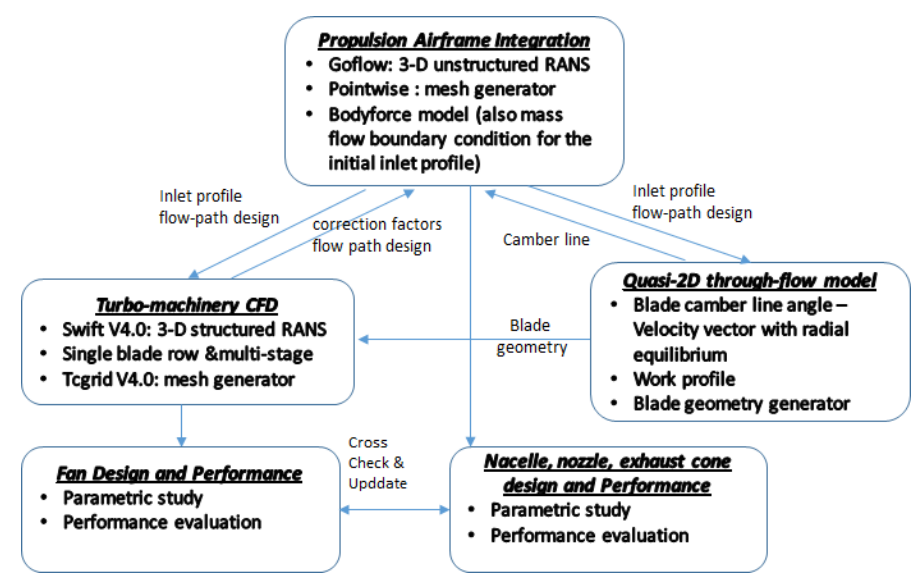

Table 2. Flow chart of the multi-fidelity propulsor design framework.

(1) To account for the radial profile of the ingested inflow, the boundary layer profile is acquired from PAI CFD with 1-D mass flow boundary condition at the AIP.

(2) With the profile, corrected speed/flow $\left(N_{\mathrm{c}}, \dot{\mathrm{m}}_{\mathrm{c}}\right)$ and given flow-path information, a quasi-2D model is constructed (input). 
(3) The work profile is defined by parametric studies in the through flow model with a constraint of the diffusion factors. If there is a fully automated geometry tool, or if the work profile can be manipulated by control points, an optimizer like NSGA-II ${ }^{19}$ could be coupled and a full optimization work will be conducted. In this study, the equation based work profile for general forced vortex types is applied and the constraint are decided by empirical models.

(4) Once the geometric definitions of the fan and EGV are obtained from low-fidelity analysis and design, the flow-path and blade/vanes are gridded with TCGRID ${ }^{14}$ and analyzed via SWIFT to validate the flow turning angle prediction from quasi-2D and evaluate the correction factors for the bodyforce model. Also the parametric studies for fan/EGV geometries are performed with SWIFT.

(5) With the calculated correction factor, a Goflow analysis is run with body-force defined by the fan/EGV geometry, loss correction factors from low fidelity models. The flow-path is finally designed by investigating the wake recovery factor, propulsive efficiency, mass flow rate, and thrust.

\section{RESULTS \& DISCUSSIONS}

Before working on the geometric design of the TCT propulsion system, an existing GE-R4 fan is used to validate our CFD-based design hypothesis in section (I). The newly proposed quasi-2D through-flow and body force models are validated with SWIFT code CFD results on a forced vortex fan shown in section (II). After the validations, the flow-path, fan, EGV and nozzle/exhaust cone are redesigned from the baseline geometry to realize the goal of the fuel saving by using the multi-fidelity framework, shown in sections (III) and (IV). Following the redesigned propulsion system, the fan/EGV performance metrics are evaluated at the operating conditions in (V). Finally, the performance of the PAI is evaluated and presented in (VI).

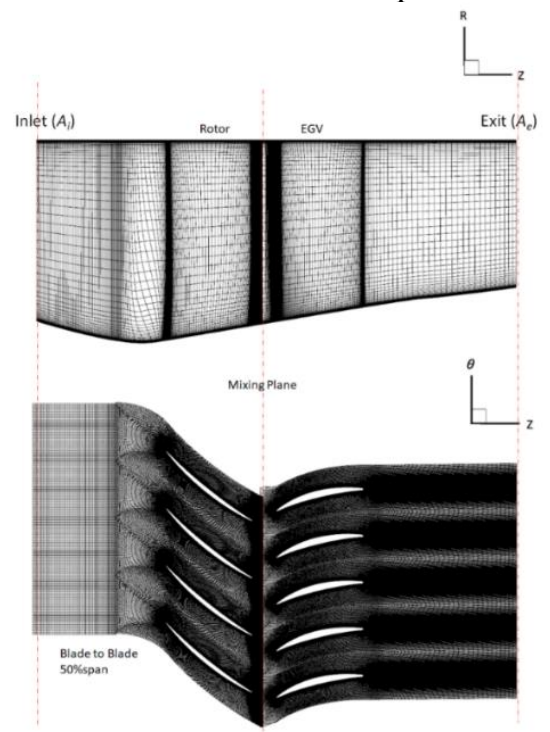

Figure 12. The mesh generation from TCGRID V4.0 for SWIFT CFD analysis. (blade to blade view mesh is scaled by 3 along the circumferential direction)
(I) Effect of the Boundary Conditions: The propulsor design in PAI applications will be more versatile if it is not boundary or operating conditions dependent. Especially, if the potential flow field of the fuselage is not affected even when the fan operates at different mass flow rates, then, the aerodynamic drag is not changed within the range of the normal fan operation.

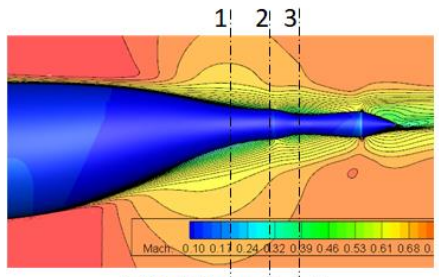

(a) No Tail-cóne Naçelle

(c) Bodyforce Fan Modeling (MFR $=163 \mathrm{~kg} / \mathrm{s}$ )

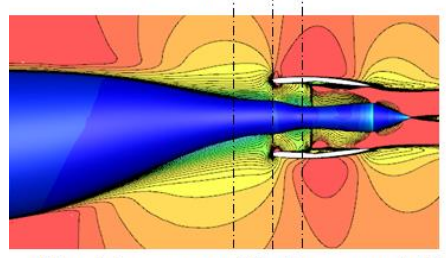

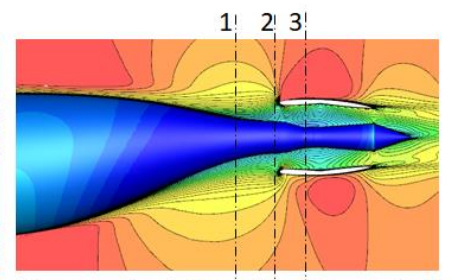

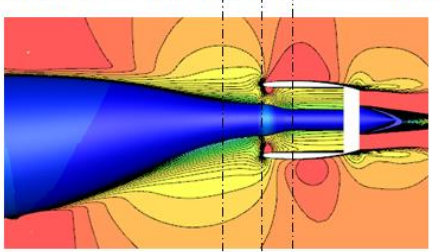

(d) Back pressure $B C(M F R=178 \mathrm{~kg} / \mathrm{s}$ ) (b) No Propulsor (through flow, MFR=129kg/s)

Figure 13. The effect of the fan operation toward the upstream flow pattern - Comparison of the Mach contours

However, it was reported ${ }^{15,21}$ that a constant static pressure (back-pressure) boundary condition, which is widely used in the 1-D engine modeling, may not valid in simulating highly distorted onset flow at the fan face. Consequently, the authors feel that the effect of the boundary condition for the upstream boundary layer profile and potential flow field should be rigorously checked before the actual discussion of the propulsor design.

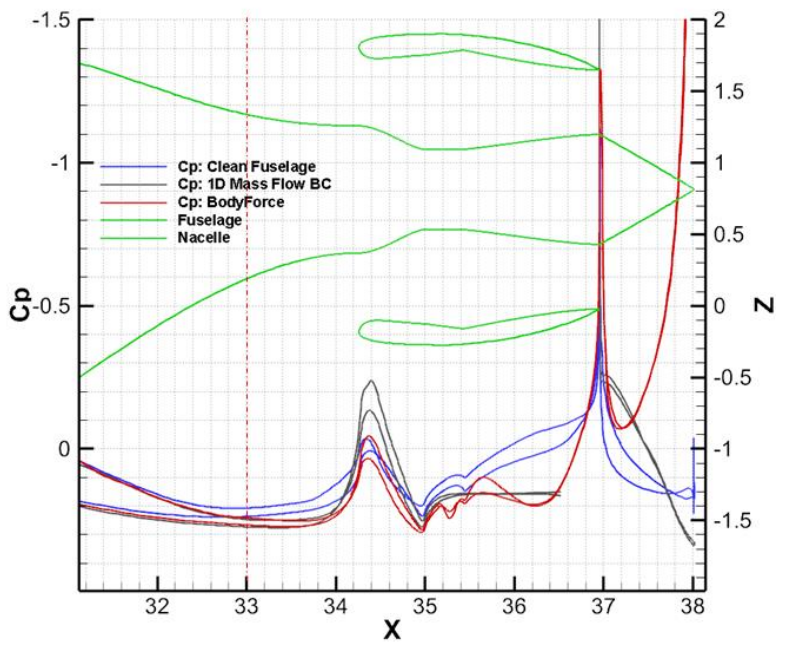

Figure 14. The effect of the fan operation on the surface pressure distribution. (The coordinate system in the figure is Cartesian.)

Figures 13 show the Mach contours of 4 cases of PAI models as follows; (a) no tail cone propulsor (i.e., no nacelle) (b) with nacelle but no actuation of the rotor (c) with nacelle and powered 
by the body-force fan actuator of the GE-R4 model (d) static pressure specified at the propulsor inlet. The static pressure condition is from the system analysis performed by $\mathrm{NPSS}^{7}$ which uses 1-D engine model. The comparison between the tail-cone configuration without nacelle case in Fig. 13-(a) and the nacelled cases in (b), (c) and (d) clearly indicates the effect of the flow blockage caused by the nacelle. However, the configuration with the actuation of the fan in Fig. 13-(c) and the through flow case in Fig. 13-(b) show almost identical flow pattern at the station 1 (location as indicated in Fig. 1) and its upstream. Only the local stream tube which are ingested into the engine between station 1 and 2 are affected by the mass flow rate. Figure 13-(d) shows the effect of the boundary condition with a constant static pressure. The same static pressure at the flow-exit boundary (AIP), but the mass flow rate is predicted much higher than that in the case (c). As a result of the higher mass flow rate, the inlet chokes at the throat while the body-force model doesn't. The external flow pattern at the nacelle shroud is also affected by the mass flow rate. However, the boundary layer characteristics at the upstream of the station 1 is not much affected even by the flow blockage of the nacelle. This also can be observed clearly in Fig. 14. The figure shows the surface pressure coefficient distribution along the axial direction with the geometry and the location of the nacelle. The case (d) indicated by the black line shows excessive expansion at the inlet throat with the same back pressure as in the case (c) in red line. Interestingly, the surface pressure distribution at the upstream of the station 1 , indicated by the vertical dashed line, is almost same for (c) and (d) cases. Even though case (a) shows a very small deviation at the upstream of the station 1 , it is local and affect the aerodynamic forces marginally. Figure 15 shows that only the case (d) predicts the boundary layer much taller than other models and the cases (a) (c) show that the boundary layer thickness on the fuselage at certain point of upstream is not much affected by the engine operation. Thus, both the viscous, and potential flow field on the surface of the airframe is not critically dependent on the propulsor design.

Accordingly, the aerodynamic coefficients such as lift and drag can be deduced to be barely changing per the fan operation at the upstream of the station 1. On the other hand, as shown in the case (d), 1-D engine model could mislead the design due to the limitation of the numerical boundary condition. The authors see that the static pressure boundary condition with radial equilibrium may predict the potential flow field of the PAI better but that would be a CFD study which is beyond the scope of the current design work.

Per the above investigation, the assumption that designing BLI propulsor will not affect the aerodynamic performance of the airframe unless the nacelle geometry or the inlet shape are significantly changed is defended. Hence, the design of a BLI propulsion system can be decoupled from the airframe.

(II) Validation of the Numerical Models: The turning angles and the total pressure profiles from the qausi-2D and body-force models for a forced vortex type fan design $(l=3)$ are compared with the radial profiles from a single blade row CFD in Figs. 16.

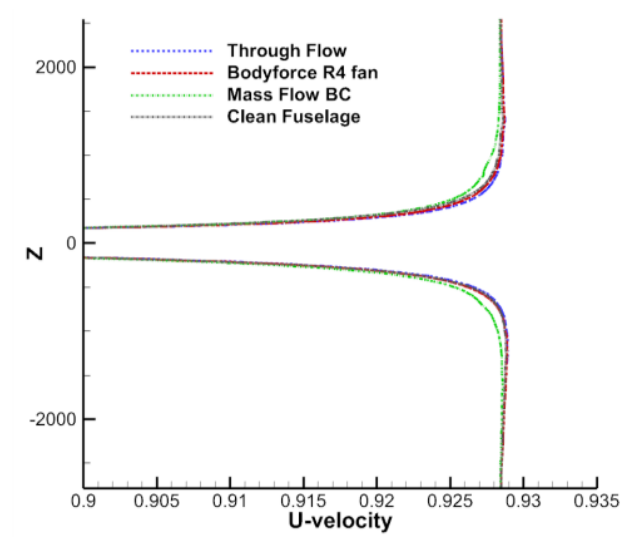

Figure 15. The effect of the fan operation toward the upstream flow pattern is displayed by the comparison of the nondimensional axial velocity.

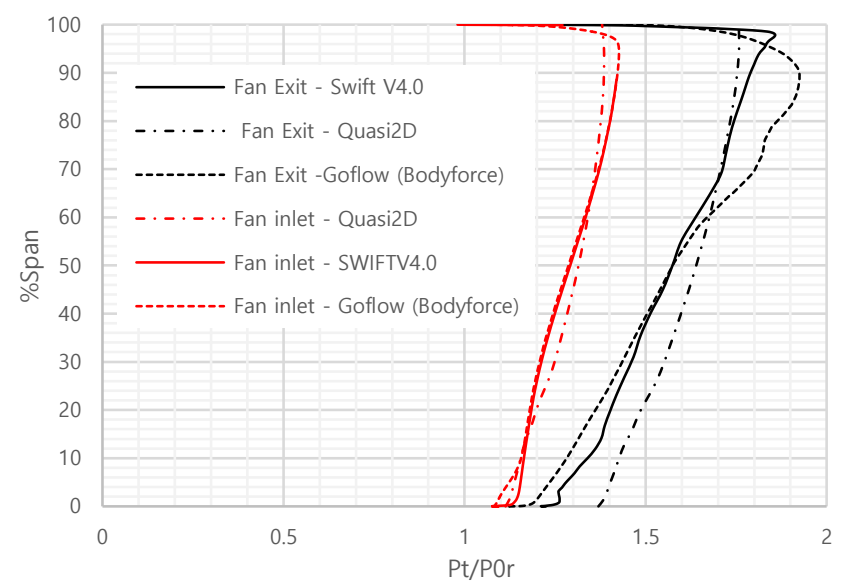

(a) Total Pressure Profiles at the AIP and Fan exit

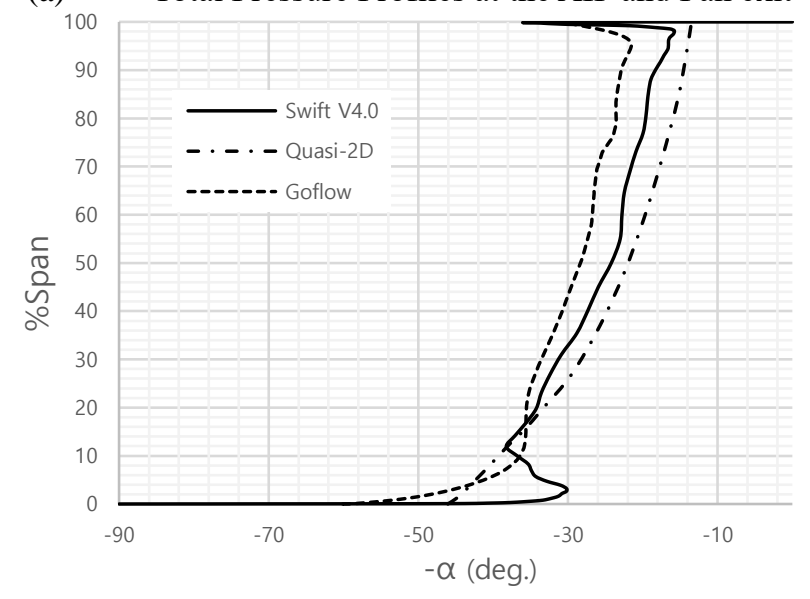

(b) Absolute Flow Angle Profiles at Fan exit

Figures 16. Fan exit profiles from the quasi-2D, PAI with bodyforce and turbo-machinery CFDs. $\left(\mathrm{N}_{\mathrm{c}}=2745 \mathrm{rpm}\right.$, $\dot{m}_{c}=665.4 \mathrm{~kg} / \mathrm{sec}, \mathrm{p} 0_{\mathrm{r}}:$ reference pressure of the air )

The correction factors in the Eq. (14) for the body-force model are adjusted to match the profiles of the flow turning, total 
pressure and temperature simultaneously. In Fig. 16-(a), the body-force model tends to under-predict the pressure rise at the low speed region near the hub while over-predict the pumping at the tip when compared with the turbomachinery CFD profile at the fan exit. Both the shapes of the profiles and the 1-D face averaged number for the total properties are matched at the respective fan and EGV exits. The quasi-2D profile predicts the total pressure more optimistic than both CFD models at the $60 \%$ span and below. This defect seems to originate from the empiricism based profile loss model which works well for the clean flow engines. As a result, the design of the metal angle in the low momentum regime is misled. Thus, to compensate the deficit of the turning, we added a linear artificial deviation angle profile in the blade generator input. The numerical turning in the quasi-2D model is based on empiricism for now, but more rigorous studies based on theoretical analyses are needed in the future work. On the other hand, the body force predicts the flow over-turning for a matched pressure profile as shown in Fig. 16(b). Since the total pressure and efficiency are more critical for the performance evaluation at the downstream of the EGV turning, the profiles of the total properties need to be matched with a higher priority.

(III) Nacelle and internal flow-path design: The specification of the geometry and the operating condition from the aircraft system design is provided in table 1. In baseline geometry, the height of the inlet highlight from the hub is $\mathrm{h}_{i}=0.54 \mathrm{~m}$; the fan diameter is $1.83 \mathrm{~m}$; the hub to tip radius ratio is 0.3 ; and the nozzle throat area is $1.73 \mathrm{~m}^{2}$. The length of the exhaust cone is $1.06 \mathrm{~m}$, and the angle of the cone is $20^{\circ}$.
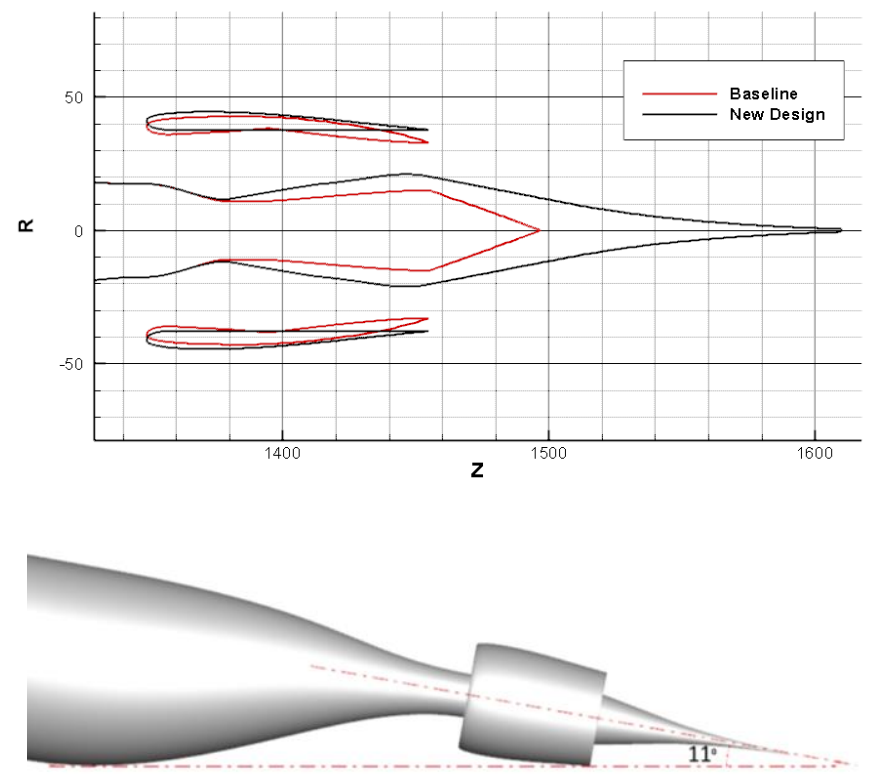

Figure 17. Flow-path, nacelle and exhaust cone design.

The inlet highlight is radially extended by $0.05 \mathrm{~m}$ in the new design to capture more flow into the engine. Fan diameter is slightly increased $(0.02 \mathrm{~m}$ at the fan face). To allow hub flowpath contraction as much as possible, the shroud of the nacelle was de-cambered as much as the tail-strike constraint at 11 degree of the angle of attack allows. The exhaust cone is concaved with a reflex point at the nozzle throat and the length is stretched 115 inches longer from the baseline to reduce the cone angle and mitigate the excessive expansion of the nozzle jet flow. The internal flow-path and fuselage, nacelle geometries designed by the NPSS model (baseline) is compared with the new flow-path design in Fig. 17. The performance comparison will be presented after iterative design between fan/EGV and the PAI models in the Chapter (VI).

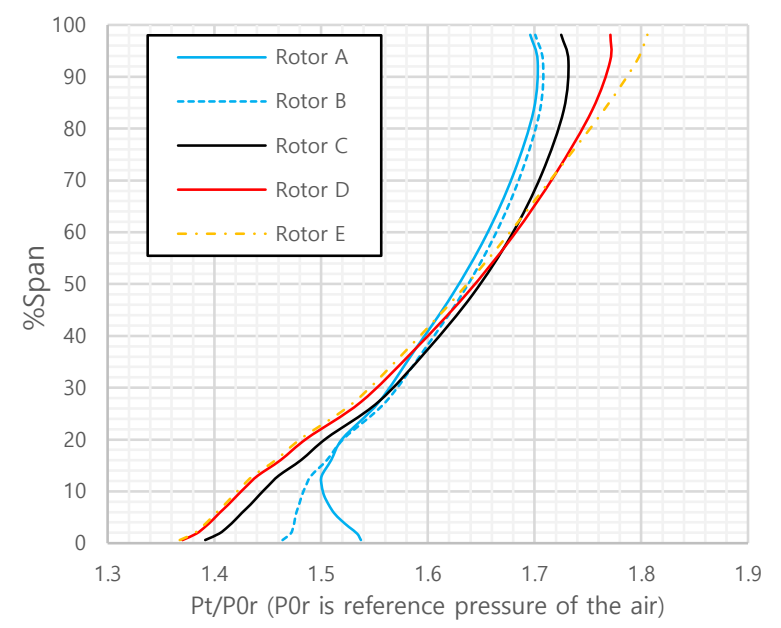

Figure 18. Fan exit profiles from the quasi-2D for parametric study by the vortex type. $\left(\mathrm{N}_{\mathrm{c}}=2745 \mathrm{rpm}, \dot{m}_{c}=665.4 \mathrm{~kg} / \mathrm{sec}\right.$, $\mathrm{FPR}=1.22$ )
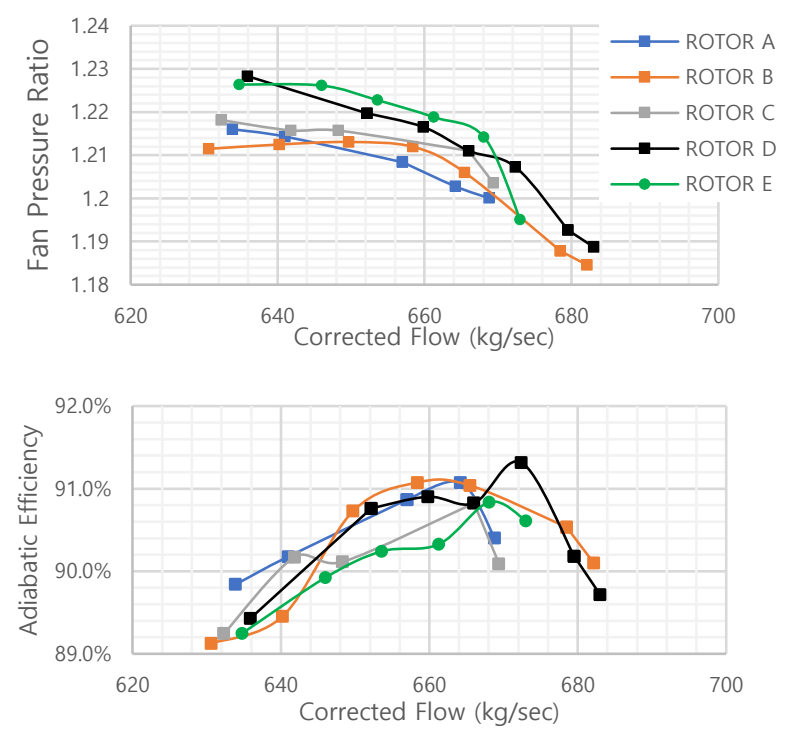

Figures 19. Speedline of the conceptual rotors for different vortex types. $\left(\mathrm{N}_{\mathrm{c}}=2745 \mathrm{rpm}, \dot{m}_{c}=665.4 \mathrm{~kg} / \mathrm{sec}, \mathrm{FPR}=1.21\right.$, Single blade row CFD, Upper: FPR vs corrected flow, Lower:

Efficiency vs corrected flow)

(IV) Fan design: The design requirements of the propulsor are the thrust of $\mathrm{T}=14.7 \mathrm{kN}$ and the shaft power of $\mathrm{P}=2610 \mathrm{~kW}$ at the 
cruise speed (at 37,000 ft. altitude). ${ }^{7}$ The rotational speed and FPR are adjusted to meet the two major requirements to realize the fuel burn saving in the CFD analysis. As the fan diameter is extended, the mass flow rate and shaft power to keep same fan pressure ratio are increased. Thus, the rotational speed is reduced to match the tip speed. The target fan pressure ratio ( $\mathrm{FPR}=1.22$, lowered from 1.25) is reduced to keep the shaft power lower than the requirement.

The quasi-2D analyses at the given design condition generate the camber angles of the fan and EGV airfoils for different vortex types $(l=-5,-3,0,3,5)$ respectively. Each type of rotor is denoted as rotor A E per the power coefficients from $l=-5$ to 5 , in the alphabetical order. The quasi-2D profiles of the non-dimensional total pressure at the fan exit of each rotor are presented in Fig. 18. The power of radius controls the radial pressure distortion and swirl of the flow toward the leading edge of the EGV. An optimal efficiency for the design condition among rotors considered could be found. The number of blade are set as 25 counts for the profile study. Figure 19 shows the fan performance of rotors along the speed line at $95.2 \% \mathrm{~N}_{\mathrm{c}}$ via CFD analyses. Overall, all tested rotors have peak efficiency point near $\dot{m}_{c}=665 \mathrm{~kg} / \mathrm{sec}$.

The $l>0$ cases show higher pressure ratio but achieved lower efficiency at the near stall conditions. The efficiency of $l<0$ cases is higher than the other group but pressure ratio is lower. It is notable that the pressure rise along the speed line is not very stable. Thus, it can be deduced that the BLI rotors show nonlinear reaction which is related to the radial distribution of the mass flow as such the compressor blades do at part-speed. The flow patterns of rotors $\mathrm{A}(l=-5), \mathrm{D}(l=3)$ and $\mathrm{E}(l=5)$ are compared in Figs. 20 at the peak efficiency points of rotors A and D. Both rotors show similar efficiency but have different pressure ratio. The rotor $\mathrm{E}$ which has low efficiency for the similar pressure rise is used as a reference to make the contrast standout. Figure 20(a) compares the stagnation pressure contours at $95.4 \%$ span near the tip. As the rotor turns more flow at the tip, the size of the shock induced separation gets larger at about $30 \%$ chord of the suction side. Thus, the loss from the wake increases from rotor A to $\mathrm{E}$. On the other hand, the rotor works more effectively as more flow goes through the hub in the comparison between rotors $\mathrm{A}$ and E in Fig. 20-(b). However, the hub diffusion of the rotor A is excessive so the profile loss is higher than that of rotor $\mathrm{D}$ per given solidity. Consequently, the pressure rise of the rotor D is the best with the highest efficiency. The current conceptual design adopts $l=3$ profile for the rest of the paper.

The chosen rotor $\mathrm{D}$ is tested for different blade counts from 16 to 34 to check the effect of the solidity. The figures 21 present the adiabatic efficiency, mass flow rate and solidity versus the blade counts (NB). Also shown is the pressure ratio and adiabatic efficiency against the hub solidity. As the hub solidity increases from 3 to 4 , the efficiency tends to increase as seen in the middle figure. The high solidity helps the flow attachment at the tip as well. Thus, the overall efficiency is improved. Due to the flow separation, the reaction of the rotor is not so consistent that the performance of some points show over- or undershoot depending on the flow blockage (as indicated by the corrected mass flow rate in the bottom figure) at the hub where the solidity is between $3 \leq \sigma_{\text {hub }}<4$ which also corresponds to $21<\mathrm{NB}_{f}<28$ as shown in the top figure. In these cases, the flow separation is observed at 50\%span and below as shown in Fig. 22-(a) at $\mathrm{NB}_{\mathrm{f}}=20$.

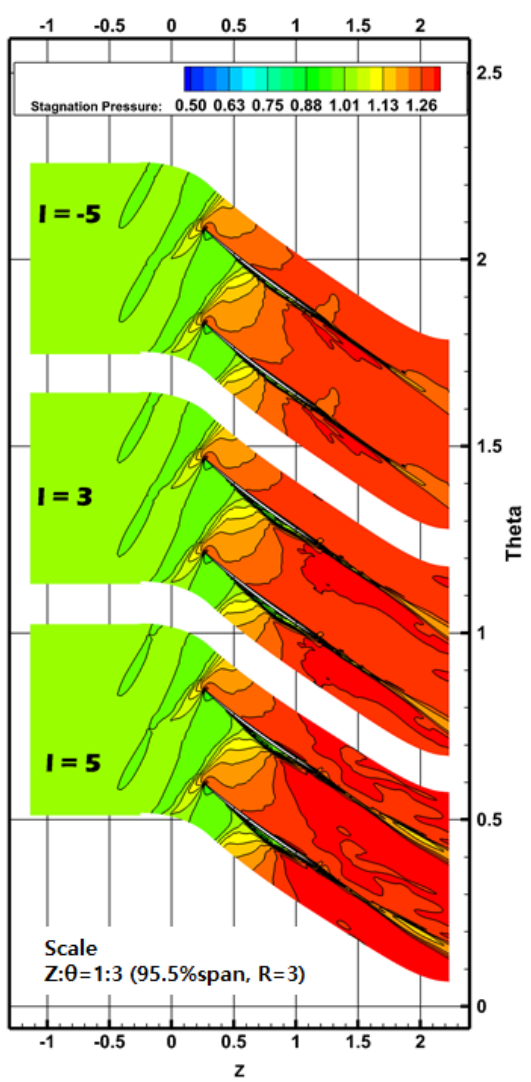

(a) Stagnation Pressure contour comparison at 95.6\%span

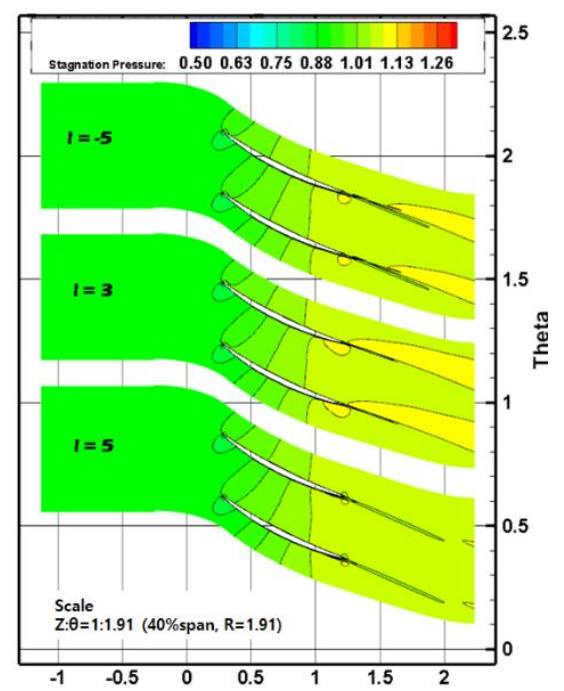

(b) Stagnation Pressure contour comparison at $40 \%$ span

Figures 20. Parametric study of the work profile. (From the top, $l=-5,3$ and 5 represent rotors A, D, and E respectively, $\mathrm{N}_{\mathrm{c}}=2745$ $\mathrm{rpm}, \dot{m}_{c} \cong 660 \mathrm{~kg} / \mathrm{sec}, \mathrm{FPR}=1.215$ ) 


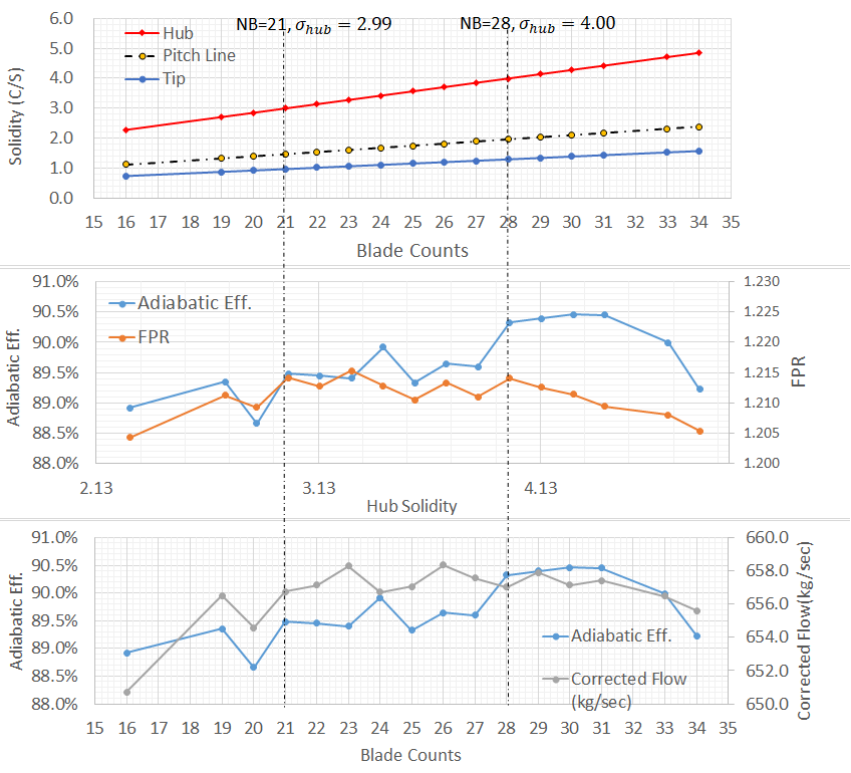

Figures 21. Parametric study for the solidity of rotor D. $\left(\mathrm{N}_{\mathrm{c}}=\right.$ $2745 \mathrm{rpm}, \dot{m}_{c} \cong 656 \mathrm{~kg} / \mathrm{sec}, \mathrm{FPR}=1.215$ )

During the transition from the low to high solidity rotors, the fan pressure ratio and the mass flow rate remain at about a constant value of 1.212 and $655 \mathrm{~kg} / \mathrm{sec}$ in average. As the hub solidity goes above 4, the reaction of the rotor changes remarkably. In Fig. 22-(a), as the hub separation is removed by increasing the solidity to $\mathrm{NB}_{\mathrm{f}}=30$, the flow blockage at the hub is minimized and shows stable wake pattern. Thus, the flow going to the tip is reduced and stabilized as shown in Fig.22-(b). As a result, there is a significant jump of efficiency while the fan pressure ratio started being reduced as the blade count increased above 28 counts as shown in Figs.21. The correlation between the generation of the loss and radial mass flow rate pattern is very similar to what is observed in the work profile comparison in Figs.19.

As the radial profile of the efficiency is observed to be strongly affected by the local solidities at the spanwise locations, the performance of the rotor $\mathrm{D}$ with different taper ratios are compared in Figs. 23. The blade count is set at $\mathrm{NB}_{\mathrm{f}}=29$ where the efficiency is near the peak while the fan pressure ratio still remains about the targeted value in the solidity study. The chord length at the pitch-line is kept constant, thus, the hub solidity increases while the tip solidity is diminished as the taper ratio decreases. High taper ratio blades with ratio between $0.8 \sim 1.0$ show higher efficiency than the lower taper ratio blades. Most of all, Fig. 23 shows that the rotor $D$ with the $T R=0.95$ blades has the best overall performance than other blades in the all tested operating speeds. Figure 24 compares the radial pressure ratio profiles of the $\mathrm{TR}=1.0,0.95,0.8$ and 0.5 rotor $\mathrm{D}$. The tip reaction of the $\mathrm{TR}=0.95$ rotor is higher than $\mathrm{TR}=0.5$ rotor. However, the pressure rise at the hub region to $60 \%$ span is healthier in $\mathrm{TR}=0.5$ rotor. The shroud region needs to hold the solidity as the level of the $\mathrm{TR}=0.95$ rotor but hub region seems to need higher solidity to keep the pumping characteristics at the high speed conditions.

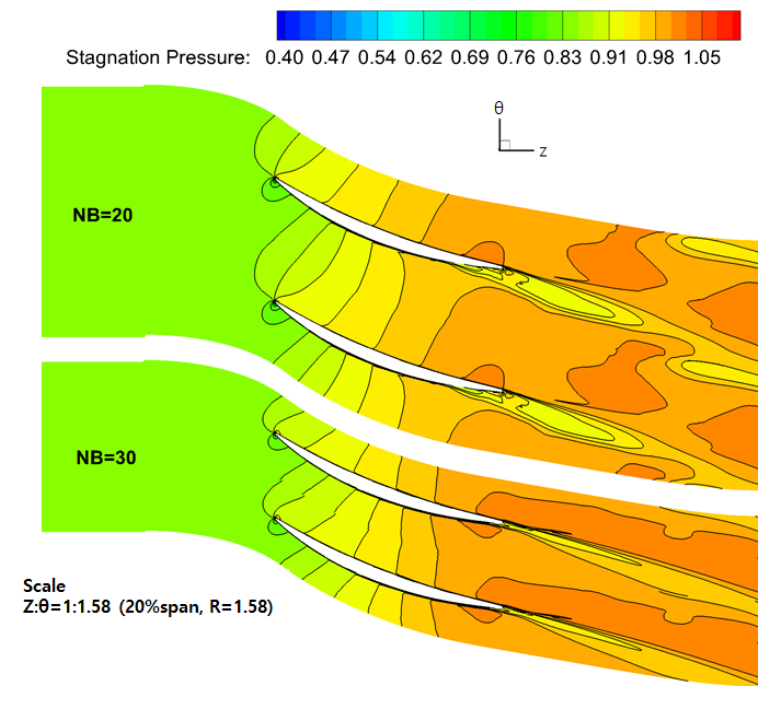

(a) Stagnation Pressure contour comparison at 20\%span

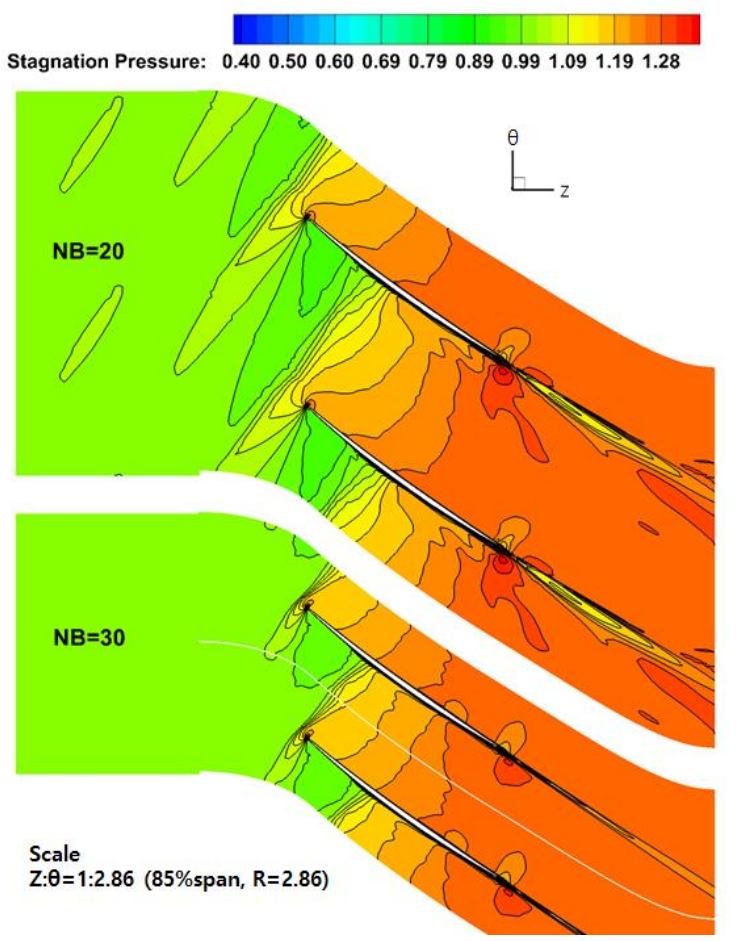

(b) Stagnation Pressure contour comparison at 85\%span

Figures 22. Parametric study of the blade counts. (From the top, $\mathrm{NB}=20$ and $30 . \mathrm{N}_{\mathrm{c}}=2745 \mathrm{rpm}, \dot{m}_{c} \cong 660 \mathrm{~kg} / \mathrm{sec}, \mathrm{FPR}=1.215$, the stagnation pressure is normalized with respect to the freestream total pressure.)

A non-linear chord distribution along the spanwise direction might be a way to improve the performance. Furthermore, more detailed design of the blade with forward/backward sweep angles, and the shaping of the camber-line and thickness distribution will be needed to achieve higher performance of the propulsor. Other specifications of the rotor design will be left for shape optimization during the detailed design in the future work. 
The same steps as described in this section are also applied to the EGV design. The number of EGVs is given as 44 counts based on the solidity study.

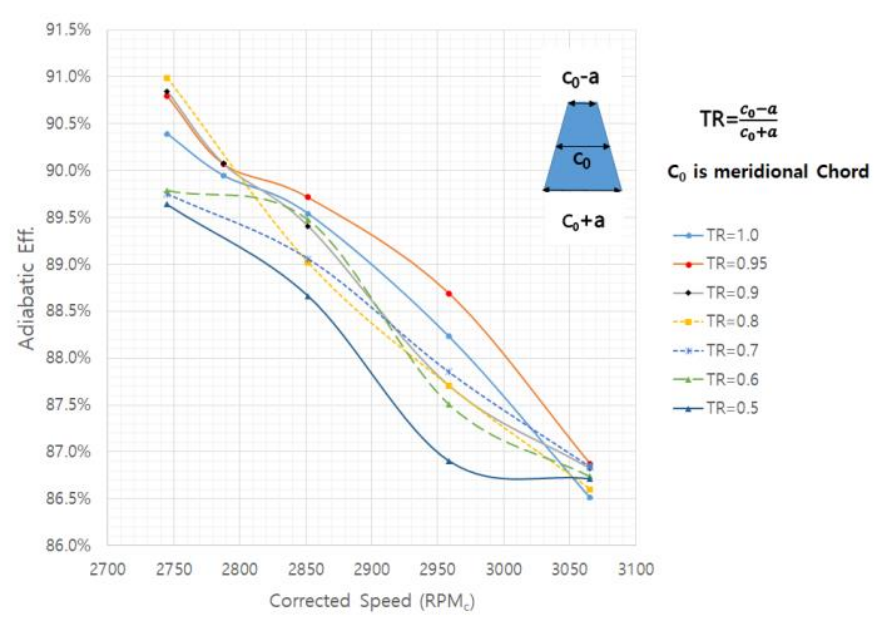

Figure 23. Parametric study for the taper ratio of the rotor D. Efficiency vs rotational speed. $\left(\mathrm{NB}_{\mathrm{c}}=29\right)$

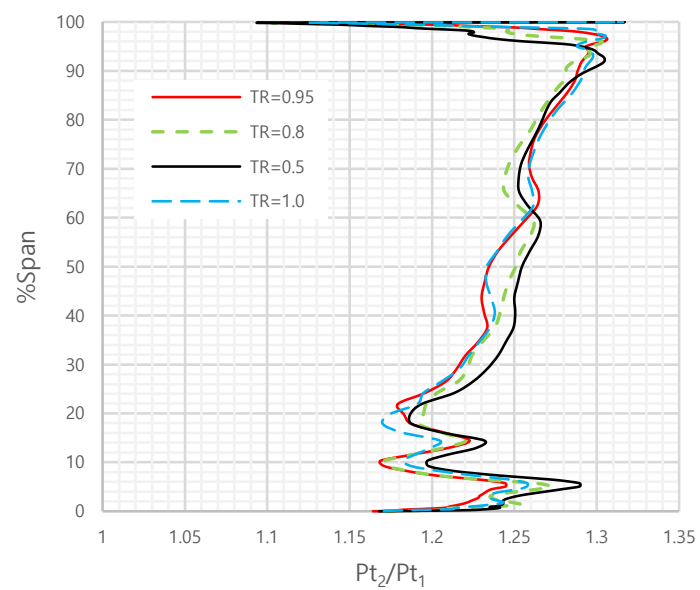

Figure 24. Total pressure ratio profile for different taper ratio. $\left(\mathrm{NB}=29, \mathrm{~N}_{\mathrm{c}}=2850 \mathrm{rpm}, \dot{m}_{c} \cong 665 \mathrm{~kg} / \mathrm{sec}, \mathrm{FPR}=1.232\right)$

(V) Fan/EGV performance: The performance map of the conceptual fan design is plotted in the Figs. 25. As the fan is designed at $\mathrm{N}_{\mathrm{c}}=2883.2 \mathrm{rpm}$, the corrected speed in Figs. 25 is expressed as a percentage of the design speed. Figure 25-(a) shows the efficiency along each speed line per the corrected flow. The peak adiabatic efficiency is observed at about $90.8 \%$ near a cruise speed of $95.2 \% \mathrm{~N}_{\mathrm{c}}$. The fan pressure ratio at the peak efficiency point is 1.212 . The peak efficiency point of $96.7 \% \mathrm{~N}_{\mathrm{c}}$ speed requires the shaft power about $2610 \mathrm{~kW}$ which meets the system design requirement at the altitude. The fan efficiency at this point is about $90.1 \%$ and the pressure ratio is 1.22 . The mass flow rate at the cruise altitude is $163.5 \mathrm{~kg} / \mathrm{sec}$. The operating and stall lines in Fig. 25-(b) is drawn per the CFD data following the peak efficiency and the pressure roll-over points of the respective speed-lines for a reference.
The radial turning profile of the fan and EGV systems at the cruise speed are presented in Figs. 26. The body-force predicts the exit flow turning reasonably well. The profiles $1 \sim 4$ denote 4 circumferential location in a counter clock-wise direction as shown in Figs. 26-(a) and (b). The turning at each location shows small deviation within 5 degree from each other and the profile shows axi-symmetric shape. Also the flow angle could be kept lower than 5 degree relative to the axial direction. The total pressure loss between LE and TE of EGV is predicted as 5.48\% relative to the fan exit total pressure based on the entropy average at the design speed $\left(100 \% \mathrm{~N}_{\mathrm{c}}\right)$.

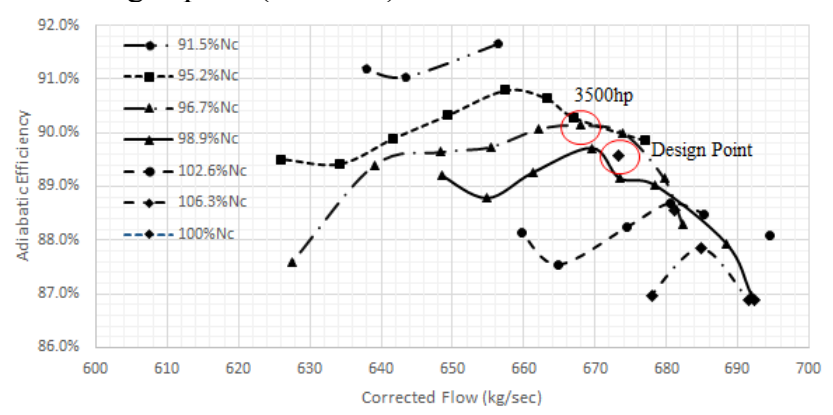

(a) Efficiency Map

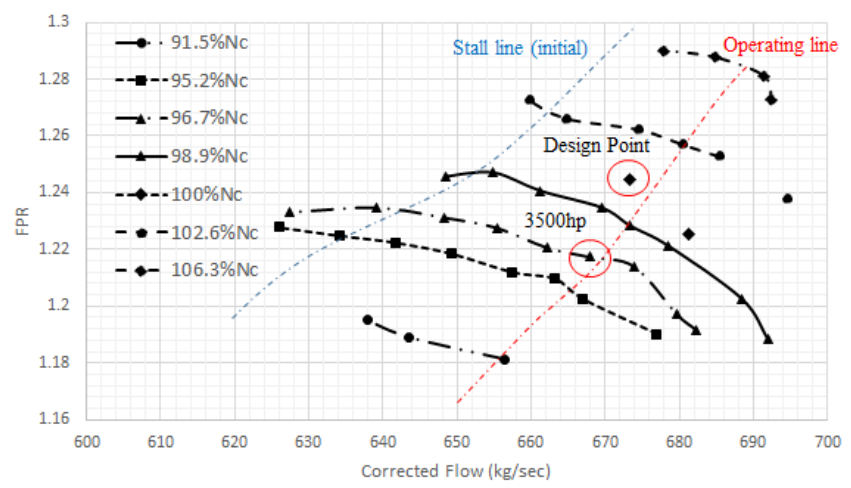

(b) Fan Pressure Ratio Map

Figures 25. Performance map of the conceptual design. ( $\mathrm{NB}=29$, Design Point: $\mathrm{N}_{\mathrm{c}}=2883.2 \mathrm{rpm}\left(100 \% \mathrm{~N}_{\mathrm{c}}\right), \dot{m}_{c} \cong 670 \mathrm{~kg} / \mathrm{sec}$, $\mathrm{FPR}=1.25$ )

(VI) PAI performance and jet profile: We have evaluated the performance of the propulsion airframe integration of the tailcone thruster with a body-force model. The correction factors of the model are derived by matching it with the CFD profiles. Figure 27 presents the thrust versus the fan pressure ratio along the operating line of the tail-cone thruster system. The two linear thrust lines are the ideal lines for the reference of the current design. The gray line indicates the system design requirements and the black line is predicted by a simple disk actuator model which adds the axial momentum via FUN3D CFD code. ${ }^{26}$ The black line is the fan pressure ratio and the computed thrust between the inlet and nozzle exit without considering losses from the operation of fan and EGV, e.g., a reversible propulsor. Thus, the line represents the maximum thrust that the baseline propulor can achieve. The performance of the new design which has an 
extended fan diameter predicted by both the fan/EGV and PAI CFD models are in a good agreement with each other. In addition, both models generate higher thrust than the ideal thrust line of the baseline at the same fan pressure ratio until the PAI model chokes at about FPR $=1.28$ while the turbo-machinery model still does not. The mass flow correction factor in the bodyforce model then has to be adjusted at the higher power regime to prevent the early choking. The thrust estimation from the turbo-machinery CFD is only about $11.2 \mathrm{kN}$ for a $2610 \mathrm{~kW}$ (3500hp) shaft power and PAI model at about $11.29 \mathrm{kN}$ while the requirement is $14.7 \mathrm{kN}$ at the same power. The curve of the thrust against the shaft power consumption as shown in Fig. 28 is plotted.

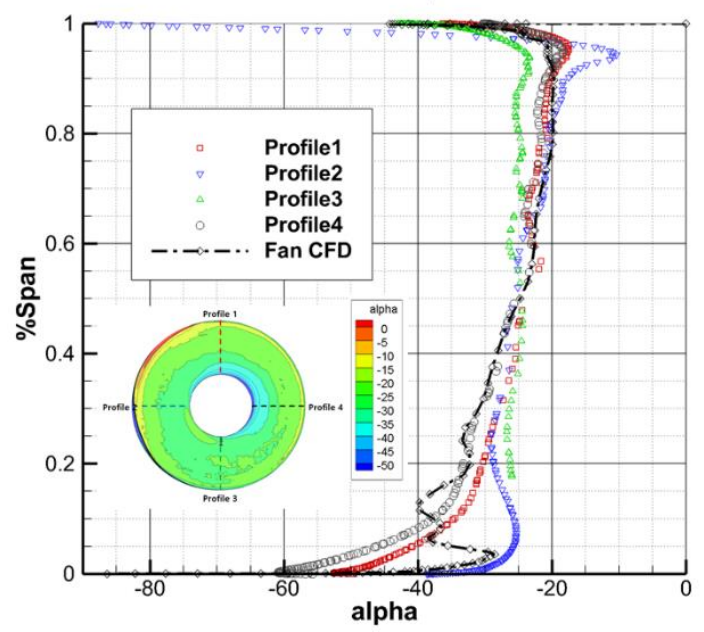

(a) Flow turning angle at the fan exit

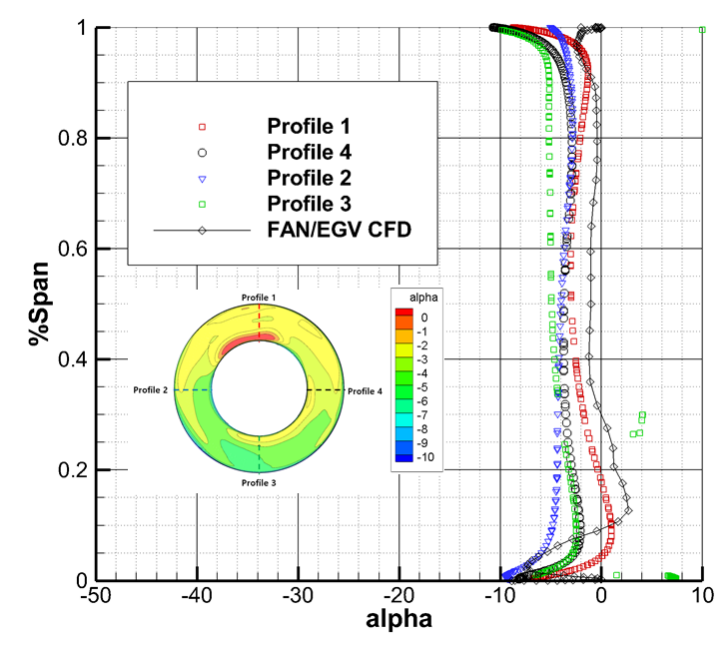

(b) Flow turning angle at the EGV exit

Figure 26. Absolute flow angle change through EGV. $\left(\% \mathrm{~N}_{\mathrm{c}}=96.7, \quad \dot{m}_{c}=665.4 \mathrm{~kg} / \mathrm{sec}, \quad \mathrm{FPR}=1.22, \quad \mathrm{NB}_{\mathrm{f}}=29\right.$, $\mathrm{NB}_{\mathrm{V}}=44$ )

The thrust-shaft power curve of the conceptual fan design $(\mathrm{FPR}=1.25)$ are compared with PAI prediction in Fig. 28. The predictions are comparable with those of the turbo-machinery model. The gray line is from the system design. The propulsor efficiency which is the instant slope of the curve shows a peak near the $3500 \mathrm{hp}$ point. The propulsive efficiency of the designed propulsor is predicted much higher than the baseline propulsor with the GE-R4 fan by about 1.2 1.3 kN near the peak efficiency region. As aforementioned the GE-R4 fan and its body-force model is used in this study as a reference of the reaction of the conventional fan in the BLI system. Note that the thrust as defined in Eq. (3) is calculated at nozzle exit which is at the upstream of the exhaust cone, thus, the loss from the exhaust cone is not reflected in the Figs. 27 and 28.

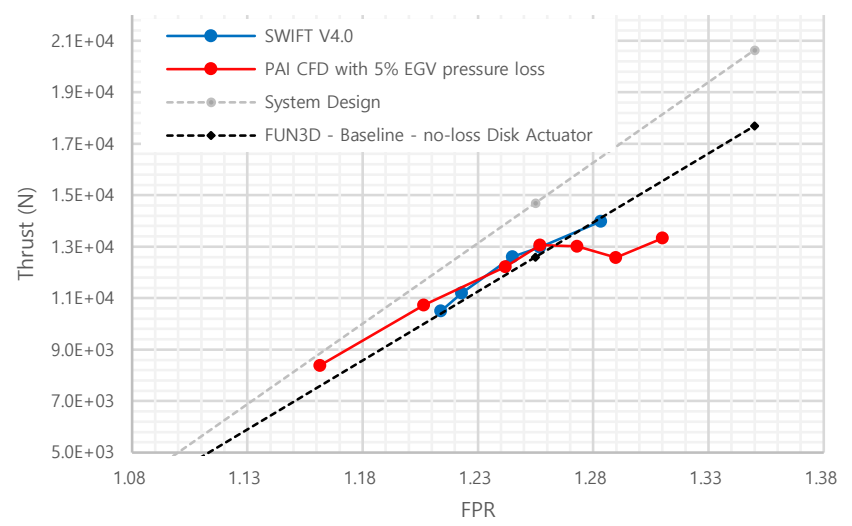

Figure 27. Thrust vs fan pressure ratio (FPR) along the operating line of the tail-cone thruster. (Note that the legends denoted as FUN3D- Baseline is the thrust line predicted by FUN3D CFD code using disk actuator model for the baseline geometry that reflects no loss from the fan/EGV operation by the courtesy of Dr. M.R.Mankbadi).

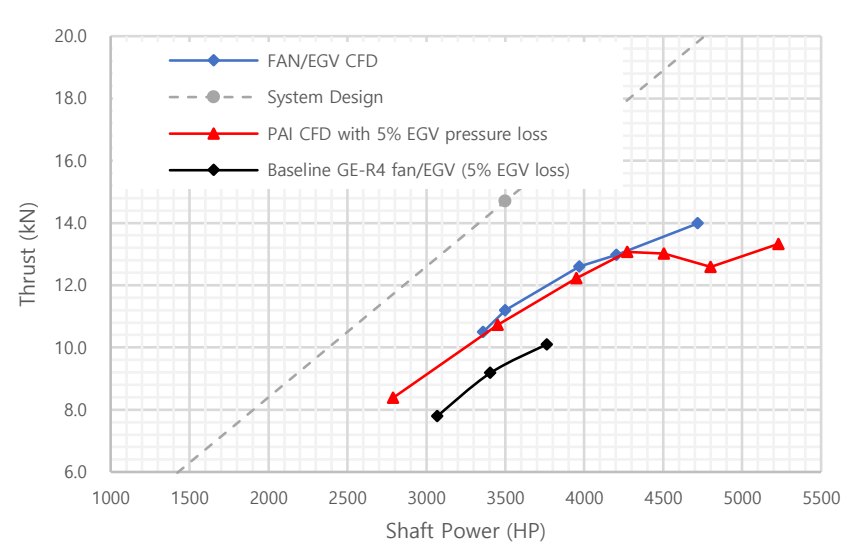

Figure 28. Thrust-shaft power curve of the designed tail-cone thruster.

Figures 29 show the entropy contours of the designed tail-cone thruster in comparison with the baseline propulsor. It reveals the sources of the loss throughout the propulsor and the wake zone downstream. The high-speed jet from the propulsor accelerates along the exhaust cone, thus, the over-expanded baseline cone causes a significant loss. On the other hand, the entropy rise of the redesigned cone is significantly lower than the baseline design for a similar shaft power condition (near $3500 \mathrm{hp}$ points). 
In addition, less cambered nacelle reduces the wake loss from the shroud trailing edge as well. Furthermore, the new design of the rotor shroud and less tip turning than conventional high transonic fan help to reduce the rotor tip loss. Figures 30 compare the jet contours and their associated total pressure losses of these two designs. The total pressure loss from the exhaust cone is quantified through a stream-tube approach by taking a circular domain that has the same mass flow rate as that of the nozzle exit, as shown in Figs. 30. The total pressure losses predicted from the stream-tubes are $2.56 \%$ (domain radius: $\mathrm{R}=0.85 \mathrm{~m}$; mass flow rate: $M F R=168 \mathrm{~kg} / \mathrm{sec}$ ) and $9.4 \%$ (domain radius: $\mathrm{R}=0.81$; $\mathrm{MFR}=132 \mathrm{~kg} / \mathrm{sec}$ ) for the new propulsor and the baseline respectively. However, the swirling jet flow is not completely concentric, thus, the circular stream-tube cannot reflect the whole variation of the total pressure in the jet flow. Hence, we compared 2 more circular domains $(\mathrm{R}=1.2 \mathrm{~m}$ and $\mathrm{R}=1.4 \mathrm{~m}$ ) until the stream-tube area gets wide enough to include all the variation of the total pressure in the jet flow for reference. The new design due to more uniformly strong jets exiting from the nozzle shows the loss is getting larger as the stream-tube includes more ambient pressure region. On the other hand, the baseline has low pressure core region which contributes most of the loss, thus, the loss gets smaller as larger stream tube is counted. (see Figs.30) The stretched cone shape reduces the low pressure region at the core remarkably as observed in Fig.30-(a).

Table 3 summarizes the performance from the PAI CFD model compared with the baseline performance and the design requirements. The total pressure loss of the baseline exhaust cone based on the entropy weighted average is about $9 \%$ relative to the nozzle exit pressure while the new design reduces the cone loss by about $70 \%$ lower based on the stream-tube of $\mathrm{R}=1(\mathrm{~m})$ at the wake. The form factor of the new inlet is 1.91 and the baseline is 1.6, thus, more benefits can be expected from the boundary layer ingestion according to Ref.[1]. The propulsive efficiency of the new propulsor design is slightly lower than the baseline but the propulsor efficiency is improved significantly. The comparison between the PAI and turbo-machinery models shows a good agreement, thus, the multi-fidelity method demonstrates the capability for the analysis and design of the boundary layer ingestion propulsor.

The adiabatic efficiency from the NPSS model is $96 \%{ }^{7}$ but the hub defect in the boundary layer keeps the rotor efficiency low at about $90 \%$. The total pressure losses of the EGV and exhaust cone contribute to those additional deficits of the propulsor performance from the design requirements. Furthermore, the accuracy of the loss predictions still cannot explain the entire reason of the thrust deficit as shown in Fig. 27. Even though the black dashed line does not account for the loss of the rotor and $\mathrm{EGV}$, it still achieves about $11 \%$ lower thrust relative to the gray line (also without considering the loss from the exhaust cone). The deficit seems to come from the ram drag, shape factor of the incoming boundary layer, and the wake recovery factor. The parameters explain the correlation between the incoming boundary layer and the performance of the propulsor which are specific characteristics of the BLI propulsion. It may not be possible to quantify these factors in an appropriate way by the system analysis methods until high fidelity numerical tools are adopted or adequate empirical models are derived from sensible experimental data for the boundary layer ingestion.

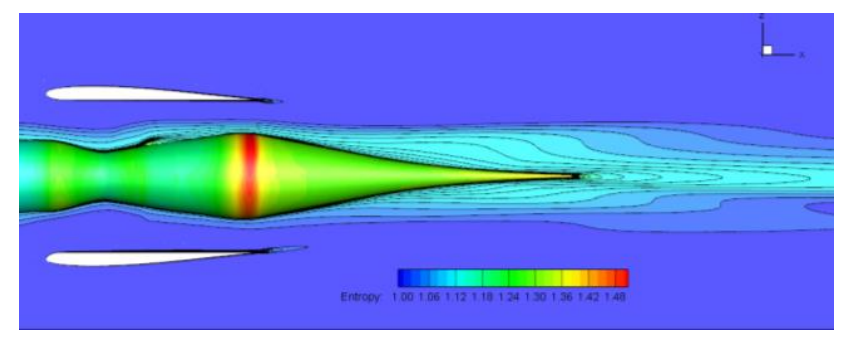

(a) New propulsor design $(\mathrm{FPR}=1.21, \mathrm{P}=3452 \mathrm{HP}$, $\mathrm{MFR}=168.7 \mathrm{~kg} / \mathrm{sec}, \mathrm{T}=11.29 \mathrm{kN})$

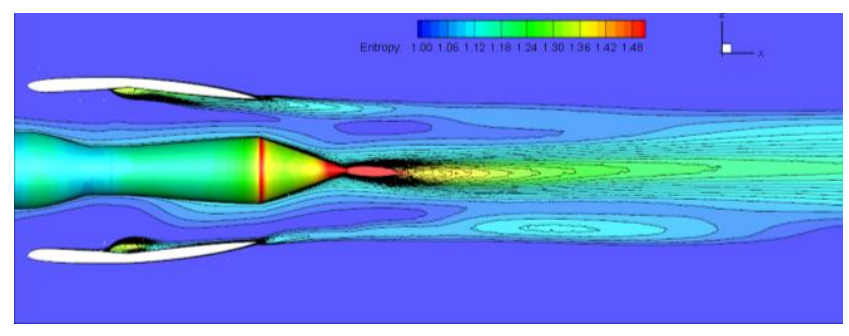

(b) Baseline propulsor $(\mathrm{FPR}=1.25, \mathrm{P}=3405 \mathrm{HP}$, $\mathrm{MFR}=132 \mathrm{~kg} / \mathrm{sec}, \mathrm{T}=9.67 \mathrm{kN}$ )

Figure 29. Entropy contours of the propulsion airframe integration of the new propulsor and the baseline designs.

\section{CONCLUSION}

The present multi-fidelity conceptual design approach for a tail-cone thruster system has successfully demonstrated the accuracy and applicability of the proposed quasi-2D method and the body-force model for the design of a BLI propulsor which is under an axi-symmetric inlet distortion. This paper describes the aerodynamic design methodology, multi-fidelity design framework and propulsor system design for a BLI aircraft. In the development of the framework, the adjustment of the correction factors of the body-force model is shown how to realize an accurate assessment of the BLI engine performance prediction in the PAI aspect. In addition, its profiles and performance are very comparable with the 3-D turbo-machinery CFD results. The proposed quasi-2D model is proved to be robust and highly efficient to generate the rotor and stator geometries at the design points under BLI inlet conditions. In the tail-cone thruster system design, the fact that the present conceptual design could not meet the design requirements indicates that the applied 1-D engine model might not be adequate for predicting the performance of the BLI propulsor. Consequently, the estimated benefits in the fuel burn saving may not be realizable. Even though it turns out that the targeted thrust could not be achieved, the accuracy of the applied models is thoroughly validated and the current work has demonstrated the capability for the future BLI fan and propulsor designs. 


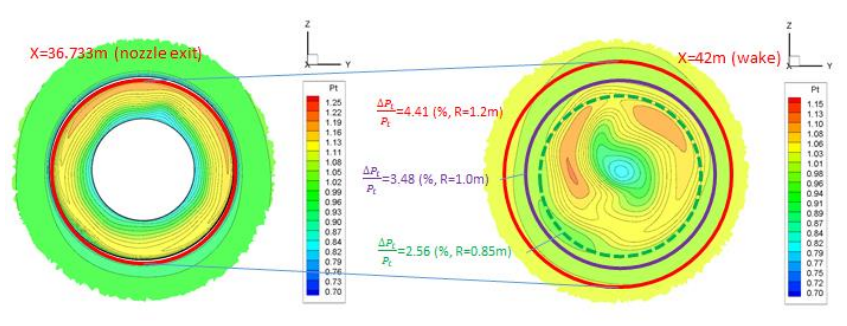

(a) New propulsor design ( $\mathrm{FPR}=1.21, \mathrm{P}=3452 \mathrm{HP}$, $\mathrm{MFR}=168.7 \mathrm{~kg} / \mathrm{sec}, \mathrm{T}=11.29 \mathrm{kN}$ )

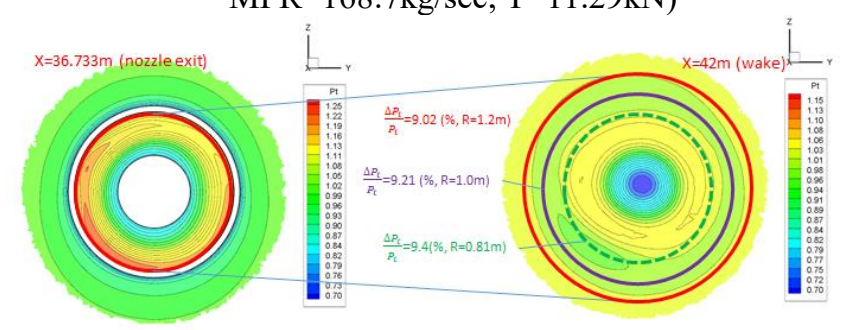

(b) Baseline Propulsor ( $\mathrm{FPR}=1.25, \mathrm{P}=3405 \mathrm{HP}$, $\mathrm{MFR}=132 \mathrm{~kg} / \mathrm{sec}, \mathrm{T}=9.67 \mathrm{kN}$ )

Figure 30. Stream-tube analysis for the measurement of the nondimensionalized total pressure loss from the exhaust cone. (Note that the contour level for each location is different)

\begin{tabular}{|c|c|c|c|c|}
\hline $\begin{array}{c}\text { Performance } \\
\text { Metrics }\end{array}$ & PAI & $\begin{array}{c}\text { Turbo- } \\
\text { machinery }\end{array}$ & $\begin{array}{c}\text { System } \\
\text { design }\end{array}$ & $\begin{array}{c}\text { Baseline } \\
\text { GE-R4 }\end{array}$ \\
\hline Form Factor & 1.91 & 1.91 & N/A & 1.6 \\
\hline MFR (kg/sec) & 168.7 & 163.5 & 157.08 & 131.8 \\
\hline Thrust (kN) & 11.29 & 11.16 & 14.7 & 9.67 \\
\hline $\begin{array}{c}\text { Propulsive power } \\
(\mathrm{kW})\end{array}$ & 1093 & 1188 & N/A & 881.2 \\
\hline Shaft power (kW) & 2574.7 & 2606 & 2610 & 2539.4 \\
\hline $\begin{array}{c}\text { Propulsive } \\
\text { Efficiency }\end{array}$ & 2.27 & 2.18 & N/A & 2.41 \\
\hline $\begin{array}{c}\text { Propulsor Efficiency } \\
(\%)\end{array}$ & 101.5 & 99.35 & N/A & 88.15 \\
\hline $\begin{array}{c}\text { Exhaust Cone Loss } \\
\left(\% \Delta \mathrm{P}_{\mathrm{t}} / \mathrm{P}_{\mathrm{t}}\right)\end{array}$ & 3.5 & N/A & 0.1 & 9.2 \\
\hline
\end{tabular}

Table 3. Performance metric of the conceptual propulsor

\section{NOMENCLATURE}

$\phi \quad$ : cone angle, exhaust cone.

$\mathrm{L} \quad$ : length of the fuselage

$\mathrm{H}_{\mathrm{i}} \quad$ : height of the inlet highlight

$\mathrm{A}_{\mathrm{c}} \quad$ : inlet captured area

AOA : angle of attack

$\mathrm{D}_{\mathrm{f}} \quad$ : fan diameter

$\mathrm{Re} / \mathrm{m} \quad$ : Reynolds number per meter

$\mathrm{T}_{\mathrm{s}} \quad$ : static temperature

$\mathrm{p}_{\mathrm{s}} \quad$ : static pressure

$\mathrm{p} 0_{\mathrm{r}} \quad$ : reference air pressure (e.g. $101.35 \mathrm{kN} / \mathrm{m}^{2}$, STD)

$\rho \quad$ : density

$\eta_{\mathrm{p}} \quad$ : propulsive efficiency

$\lambda \quad$ : quasi-normal angle

$\varphi \quad$ : flow angle between the meridional and axial directions

$\varepsilon \quad$ : numerical error in angle definition $\beta \quad$ : relative flow angle in rotational frame

$\alpha \quad$ : turning angle

$\omega \quad$ : rotational angular velocity

$\triangle \quad$ : difference

A : area

AIP : Aerodynamic Interface Plane

$\sigma \quad:$ solidity $(=\mathrm{c} / \mathrm{s})$

c : chord length

$\mathrm{s} \quad$ : pitch length

S : entropy

$r \quad:$ radius in the cylindrical coordinate

MFR : mass flow rate

FPR : fan pressure ratio

$\mathrm{m} \quad$ : meridional direction

$\dot{m} \quad$ : mass flow rate

$M \quad:$ momentum vector $\left(M_{m}=\rho W_{m}, M_{\theta}=\rho W_{\theta}, M_{n}=\rho W_{n}\right)$

$n \quad$ : normal direction to the meridional direction

$\mathrm{N}_{\mathrm{c}} \quad$ : corrected rotational speed (rpm)

$\mathrm{N} \quad$ : Physical rotational speed (rpm)

$P R \quad$ : total pressure ratio

TR : Taper ratio

$P_{p} \quad$ : propulsive power

$T \quad$ : thrust

$\mathrm{T}_{\mathrm{t}} \quad$ : total temperature

$\mathrm{P}_{\mathrm{t}} \quad$ : total pressure

$U \quad$ : tangential velocity of rotor $(\mathrm{r} \omega)$

$\overrightarrow{\mathrm{V}} \quad$ : velocity vector in cylindrical coordinates $\left(V_{z}, V_{r}, V_{\theta}\right)$

$\overrightarrow{\mathrm{W}} \quad$ : relative velocity vector in cylindrical coordinates $\left(W_{z}, W_{r}, W_{\theta}\right)$

$\mathrm{V}_{\infty} \quad$ : free stream velocity

$\mathbb{W} \quad$ : work

Subscript

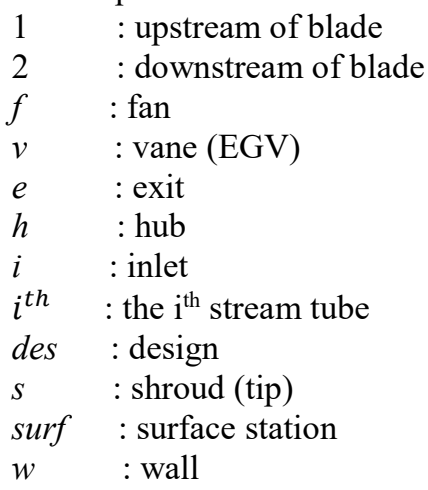

\section{ACKNOWLEDGMENTS}

In memory of the late Dr. Meng-Sing Liou, the authors sincerely appreciate our co-author and like to honor his passion and dedication to the development of the next generation subsonic aircraft with boundary layer ingestion propulsion. We also appreciate financial support of NASA's AATT project and Mr. William Haller, the technical lead of the SA\&I subproject. Special thanks to Dr. Mark Celestina (UPAI propulsion technical lead) for the discussion of the direction of propulsor design, Dr. Christopher Hughes (AATT UPAI sub-project manager) for the 
project support, Dr. Jason Welstead (TCT PI) for airframe integration of STARC-ABL and Dr. M.R. Mankbadi for FUN3D CFD analyses.

\section{REFERENCES}

${ }^{1}$ Smith, L. H., "Wake Ingestion Propulsion Benefit," J. Propul-sion and Power, Vol. 9, 74-82, 1993.

${ }^{2}$ Felder, J., Kim, H. D., and Brown, G. V., "An Examination of the Effects of Boundary Layer Ingestion on Turboelectric Distributed Propulsion Systems," AIAA-2011-300, AIAA, 2011.

${ }^{3}$ Lee, B.J., Liou, M.S. and Kim, C., "Optimizing a Boundary-LayerIngestion Offset Inlet by Discrete Adjoint Approach," AIAA J. Vol. 48, 2010, doi: 10.2514/1.J050222

${ }^{4}$ Florea, R.V., Voytovych, D., Tillman, G., Stucky, M., Shabbir, A., Sharma, O., and Arend, D., "Aerodynamic Analysis of a BoundaryLayer-Ingesting Distortion-Tolerant Fan," Proceedings of ASME Turbo Expo 2013, San Antonio Texas, GT2013-94656, June 2013.

${ }^{5}$ Cousins, W.T., Voytovch, D. and Tillman, G., "Design of a DistortionTolerant Fan for a Boundary-Layer Ingesting Embedded Engine Application," AIAA 2017-5042, 53rd AIAA/SAE/ASEE Joint Propulsion Conference, 10-12 July 2017, Atlanta, GA

${ }^{6}$ Arend, D., Wolter, J.D., Hirt, S.M., Provenza, A.J., Gazzaniga, J.A., Cousins, W.T., Hardin, L.W., and Sharma, O.P., "Experimental Evaluation of an Embedded Boundary Layer Ingesting Propulsor for Highly Efficient Subsonic Cruise Aircraft," AIAA 2017-5041, 53rd AIAA/SAE/ASEE Joint Propulsion Conference, 10-12 July 2017, Atlanta, GA

${ }^{7}$ J. R. Welstead and J. L. Felder, "Conceptual Design of a Single-Aisle Turboelectric Commercial Transport with Fuselage Boundary Layer Ingestion," AIAA-2016-1027, 54th AIAA Aerospace Sciences Meeting, San Diego, California, 2016.

8 Drela, M., "Development of the D8 Transport Configuration," AIAA2011-3970, 29 $9^{\text {th }}$ AIAA Applied Aerodynamics Conference, 27-30 June 2011, Honolulu, HI.

${ }^{9}$ Hall, D.K., Huang, A.C., Uranga, A., Creitzer, E.M., Drela, M., and Sato, Sho, "Boundary Layer Ingestion Propulsion Benefit for Transport Aircraft," J. Propulsion and Power, Vol. 33, No. 5, September-October 2017.

${ }^{10}$ Liou, M.F., Kim, H.J., B.J.Lee, and Liou, M.S., “Aerodynamic Design of Integrated Propulsion-Airframe Configuration of the Hybrid Wingbody Aircraft," AIAA 2017-3411, 35 ${ }^{\text {th }}$ AIAA Applied Aerodynamics Conference, 05-09, 2017, Denver, Co, 10.2514/6.20173411

${ }^{11}$ Kim, H.J., Liou, M.-F. and Liou, M.-S. "Mail-Slot Nacelle Shape Design for N3-X Hybrid Wing Body Configuration," AIAA 2015-3805, AIAA Propulsion \& Energy Forum 2015, Orlando, FL, 27-29 July 2015. 12 Jones, S.M., "Development of an Object-Oriented Turbomachinery Analysis Code within the NPSS Framework," NASA/TM-2014-216621 13 Aungier, R.H., "Axial-Flow Compressors: A Strategy for Aerodynamic Design and Analysis," NEW YORK ASME PRESS 2003, ISBN-13: 978-0791801925

14 Adamczyk, J., Celestina, M., and Beach, T., "Simulation of 3-D Viscous Flow Within a Multi-Stage Turbine," 34th International Gas Turbine and Aeroengine Congress and Exposition, Toronto, Canada, June 4-8, 1989.

${ }^{15}$ Chima, R. V., "Rapid Calculations of Three-Dimensional Inlet/Fan Interaction," NASA Fundamental Aeronautics 2007 Annual Meeting, New Orleans, LA, Oct. 30-Nov. 1, 2007.

16 A.Arntz, O. Atinault, D. Destarac, and A. Merlen, "Exergy-based Aircraft Aeropropulsive Performance Assessment: CFD Application to
Boundary Layer Ingestion,” AIAA 2014-2573, 32nd AIAA Applied Aerodynamics Conference, AIAA AVIATION Forum, https://doi.org/10.2514/6.2014-2573.

${ }^{17}$ Hughes, C. E., "Aerodynamic Performance of Scale-Model Turbofan Outlet Guide Vanes Designed for Low Noise," AIAA-2002-0374, AIAA, 2002. Also NASA/TM-2001-211352, 2011.

${ }^{18}$ König, W. M., Hennecke, D. K., and Fottner, L., "Improved Blade Profile Loss and Deviation Angle Models for Advanced Transonic Compressor Bladings: Part I-A Model for Subsonic Flow, and Part II-A Model for Supersonic Flow," J. of Turbomachinery, Vol 118, Issue 1, pg. 73-87, Jan 1996.

19 Deb, K. Agrawal, S., Pratap, A., Meyarivan, T., "A Fast and Elitist multi-objective Genetic Algorithm: NSGA-II," IEEE Transactions on Evolutionary Computation (IEEE-TEC), Vol.6, No.2, pp.182-197, 2002.

${ }^{20}$ Gong, Y., A, "Computational Model for Rotating Stall Inception and Inlet Distortion in Multistage Compressors," Ph.D. Dissertation, Massachusetts Institute of Technology, Dept of Aeronautics and Astronautics, 1998.

21 Kim, H.J., and Liou, M.-S. "Flow simulation and optimal shape design of N3-X hybrid wing body configuration using a body force method," Aerospace Science and Technology, Oct, 2017.

${ }^{22}$ Kenway, Gaetan, Cadieux, F. and Kiris, C. "Aerodynamic shape optimization of the STARC-ABL concept for minimal inlet distortion," submitted to AIAA SciTech 2018.

23 Defoe, J., "Inlet Swirl Distortion Effects on the Generation and Propagation of Fan Rotor Shock Noise," Ph.D. Dissertation, Massachusetts Institute of Technology, Dept. of Aeronautics and Astronautics, 2011.

${ }^{24}$ Marble, F. E., "Three-Dimensional Flow in Turbomachines," in High Speed Aerodynamics and Jet Propulsion, Vol X, Aerodynamics of Turbines and Compressors, Hawthorne, W. R. ed. Princeton University Press, Princeton, NJ, 1964, pp83-165.

25 Batchelor, G.K. (1967). An Introduction to Fluid Dynamics. Cambridge University Press. ISBN 0-521-66396-2.

26 Biedron, R. T., Derlaga, J. M., Gnoffo, P. A., Hammond, D. P.,

Jones, W. T., Kleb, B., Lee-Rausch, E. M., Nielsen, E. J., Park, M. A., Rumsey, C.L., Thomas, J. L., Wood, W. A., "FUN3D Manual: 12.4," NASA TM-2014-218179, 2014. 